\title{
HOMOGENIZATION OF PERIODIC NONCONVEX INTEGRAL FUNCTIONALS IN TERMS OF YOUNG MEASURES
}

\author{
Omar Anza Hafsa ${ }^{1}$, Jean-Philippe Mandallena ${ }^{2,3}$ And Gérard Michaille ${ }^{2,3}$
}

\begin{abstract}
Homogenization of periodic functionals, whose integrands possess possibly multi-well structure, is treated in terms of Young measures. More precisely, we characterize the $\Gamma$-limit of sequences of such functionals in the set of Young measures, extending the relaxation theorem of Kinderlherer and Pedregal. We also make precise the relationship between our homogenized density and the classical one.
\end{abstract}

Mathematics Subject Classification. 35B27, 49J45, 74N15.

Received June 4, 2004. Revised February 16, 2005.

\section{INTRODUCTION AND MAIN RESUltS}

Let $m, N \geq 1$ be two integer, $p>1, \Omega \subset \mathbb{R}^{N}$ a bounded open set with Lipschitz boundary $\partial \Omega$, and $\left.Y=\right] 0,1\left[{ }^{N}\right.$. We denote the space of all real $m \times N$ matrices by $\mathbb{M}$. Consider the integral

$$
F_{\varepsilon}\left(u_{\varepsilon}\right):=\int_{\Omega} f\left(\frac{x}{\varepsilon}, \nabla u_{\varepsilon}(x)\right) \mathrm{d} x
$$

where $\varepsilon>0, u_{\varepsilon}: \Omega \rightarrow \mathbb{R}^{m}$ and $f: \mathbb{R}^{N} \times \mathbb{M} \rightarrow[0,+\infty[$ is a Carathéodory integrand, possibly with multi-well structure, satisfying the following two conditions:

$\left(\mathrm{C}_{1}\right)$ for every $\xi \in \mathbb{M}, f(\cdot, \xi)$ is $Y$-periodic, i.e., $f(x+z, \xi)=f(x, \xi)$ for all $x \in \mathbb{R}^{N}$ and all $z \in \mathbb{Z}^{N}$.

$\left(\mathrm{C}_{2}\right) \alpha|\xi|^{p} \leq f(x, \xi) \leq \beta\left(1+|\xi|^{p}\right)$ for all $x \in \mathbb{R}^{N}$, all $\xi \in \mathbb{M}$ and some $\alpha, \beta>0$.

In pseudo ${ }^{1}$-nonlinear elasticity, when $m=N=3, F_{\varepsilon}$ in (1) is the free-energy functional at a microscopic scale $\varepsilon$ of an elastic material which occupies the bounded open set $\Omega \subset \mathbb{R}^{3}$ in a reference configuration, the body is assumed to have a periodic structure with period $\varepsilon Y$ at any scale $\varepsilon$. Roughly, following the idea of Ball and

\footnotetext{
Keywords and phrases. Young measures, homogenization.

1 Institut für Mathematik, Universität Zürich, Winterthurerstrasse 190, CH-8057 Zürich, Switzerland; anza@math.unizh.ch

2 EMIAN (Équipe de Mathématiques, d'Informatiques et Applications de Nîmes), Centre Universitaire de Formation et de Recherche de Nîmes, Site des Carmes, Place Gabriel Péri, Cedex 01, 30021 Nîmes, France;

3 I3M (Institut de Mathématiques et Modélisation de Montpellier) UMR-CNRS 5149, Université Montpellier II, Place Eugène Bataillon, 34090 Montpellier, France; micha@math.univ-montp2.fr

${ }^{1}$ We use the term "pseudo" to mean that $f(x, \cdot)$ does not satisfy the basic condition that infinite energy is needed to compress the material to zero volume, i.e., $f(x, \xi) \rightarrow+\infty$ as $\operatorname{det}(\xi) \rightarrow 0^{+}$.
}

(C) EDP Sciences, SMAI 2006 
James [4], the fine microstructure of the material can be thought of as an element of an $\varepsilon$-minimizing sequence $\left\{u_{\varepsilon}\right\}_{\varepsilon}$ for $F_{\varepsilon}$ in $\mathcal{U}$ with

$$
\mathcal{U}:=\left\{u \in W^{1, p}\left(\Omega ; \mathbb{R}^{m}\right): u=0 \text { on } \Gamma\right\},
$$

where $\Gamma$ is a subset of $\partial \Omega$ with positive $(N-1)$-dimensional Hausdorff measure. The homogenization theorem ${ }^{2}$, firstly established by Braides in [6] and then completed by Müller in [19], states that if $f$ satisfies $\left(\mathrm{C}_{1}\right)$ and $\left(\mathrm{C}_{2}\right)$ then the homogenized free-energy functional of the material in terms of Sobolev functions

$$
F_{\mathrm{hom}}(u):=\int_{\Omega} f_{\mathrm{hom}}(\nabla u(x)) \mathrm{d} x,
$$

where $f_{\text {hom }}: \mathbb{M} \rightarrow[0,+\infty[$ (the homogenized free-energy density of the material) is defined by

$$
f_{\text {hom }}(\xi):=\inf _{k \in \mathbb{N}^{*}} \inf \left\{f_{k Y} f(x, \xi+\nabla \phi(x)) \mathrm{d} x: \phi \in W_{0}^{1, p}\left(k Y ; \mathbb{R}^{m}\right)\right\},
$$

characterizes the $W^{1, p}$-weak limits of $\varepsilon$-minimizing sequences for $F_{\varepsilon}$ in $\mathcal{U}$. More precisely: $\lim _{\varepsilon \rightarrow 0} \inf _{\mathcal{U}} F_{\varepsilon}=$ $\min _{\mathcal{U}} F_{\text {hom }}$; the $W^{1, p}$-weak limit $u$ of any $\varepsilon$-minimizing sequence $\left\{u_{\varepsilon}\right\}_{\varepsilon}$ for $F_{\varepsilon}$ in $\mathcal{U}$ is a minimizer for $F_{\text {hom }}$ in $\mathcal{U}$; conversely, any minimizer $u$ for $F_{\text {hom }}$ in $\mathcal{U}$ is the $W^{1, p}$-weak limit of some $\varepsilon$-minimizing sequence for $F_{\varepsilon}$ in $\mathcal{U}$. Such a $u$ can be thought of as a "macroscopic representation" of the fine microstructure of the material.

In the homogeneous case, when $f$ does not depend on $x$ (so that $F_{\varepsilon}=F$ ), another characterization can be obtained by using the notion of gradient Young measure due to Kinderlehrer and Pedregal $[15,16]$ : a $\mathcal{W}$-gradient Young measure, with $\mathcal{W} \subset W^{1, p}\left(\Omega ; \mathbb{R}^{m}\right)$, is a Young measure $\mu$ on $\Omega \times \mathbb{M}$ for which there exists a bounded sequence $\left\{u_{\varepsilon}\right\}_{\varepsilon}$ in $\mathcal{W}$ such that $\mu$ is the narrow limit of $\delta_{\nabla u_{\varepsilon}(x)} \otimes \mathrm{d} x$ as $\varepsilon \rightarrow 0$ (cf. Sect 2.1) $)^{3}$. The relaxation theorem of Kinderlehrer and Pedregal states that under $\left(\mathrm{C}_{2}\right)$, the relaxed free-energy functional of the material in terms of Young measures

$$
\bar{F}(\mu):=\int_{\Omega}\left(\int_{\mathbb{M}} f(\xi) \mathrm{d} \mu_{x}(\xi)\right) \mathrm{d} x,
$$

where the variable $\mu=\mu_{x} \otimes \mathrm{d} x$ is a $W^{1, p}\left(\Omega ; \mathbb{R}^{m}\right)$-gradient Young measure, characterizes the weak limits of minimizing sequences for $F$ in $\mathcal{U}$ as follows: $\inf _{\mathcal{U}} F=\min _{\mathfrak{U}} \bar{F}$, where $\mathfrak{U}$ is the set of all $\mathcal{U}$-gradient Young measures; the narrow limit $\mu$ of any $\delta_{\nabla u_{\varepsilon}(x)} \otimes \mathrm{d} x$ as $\varepsilon \rightarrow 0$, where $\left\{u_{\varepsilon}\right\}_{\varepsilon}$ is minimizing for $F$ in $\mathcal{U}$, is a minimizer for $\bar{F}$ in $\mathfrak{U}$; conversely, any minimizer $\mu$ for $\bar{F}$ in $\mathfrak{U}$ is the narrow limit of some $\delta_{\nabla u_{\varepsilon}(x)} \otimes \mathrm{d} x$ as $\varepsilon \rightarrow 0$, where $\left\{u_{\varepsilon}\right\}_{\varepsilon}$ is minimizing for $F$ in $\mathcal{U}$. Moreover, $\min _{\mathcal{U}} \mathcal{Q} F=\min _{\mathfrak{U}} \bar{F}$, where

$$
\mathcal{Q} F(u):=\int_{\Omega} \mathcal{Q} f(\nabla u(x)) \mathrm{d} x
$$

(the relaxed free-energy functional of the material in terms of Sobolev functions) with $\mathcal{Q} f: \mathbb{M} \rightarrow[0,+\infty[$ the quasiconvexification of $f$ (the relaxed free-energy density of the material) given by ${ }^{4}$

$$
\mathcal{Q} f(\xi)=\inf \left\{\int_{Y} f(\xi+\nabla \phi(x)) \mathrm{d} x: \phi \in W_{0}^{1, p}\left(Y ; \mathbb{R}^{m}\right)\right\} .
$$

Finally, $u$ is a minimizer for $\mathcal{Q} F$ in $\mathcal{U}$ if and only if there exists $\mu=\mu_{x} \otimes \mathrm{d} x$ minimizer for $\bar{F}$ in $\mathfrak{U}$ such that $\nabla u(x)=\int_{\mathbb{M}} \zeta \mathrm{d} \mu_{x}(\zeta)$ for a.e. $x \in \Omega$. Such a $\mu$ can be thought of as a "microscopic representation" of the fine microstructure of the material.

\footnotetext{
2 In the convex case, the homogenization theorem was proved by Marcellini in [18].

3 To simplify the presentation of the paper, we will denote by $\mathrm{d} x$ the Lebesgue measure restricted to any bounded open subset of $\mathbb{R}^{N}$.

4 The quasiconvexification formula was established by Dacorogna in [10].
} 
In our paper we extend the relaxation theorem of Kinderlherer and Pedregal to the periodic homogenization by means of a $\Gamma$-convergence procedure (for an other approach about $\Gamma$-convergence through Young measures, we refer to [21]). In the classical homogenization process, gradient solutions of $\min _{\mathcal{U}} F_{\text {hom }}$ capture the oscillations due to the periodic structure. Unfortunately, as the density $f_{\text {hom }}$ is quasiconvex, we loose the information about the oscillations developed by the gradient minimizing sequences because of the multi-well structure. By considering our process, every probability solution of the new limit problem captures two kinds of oscillations: those due to the $\varepsilon$-periodicity (by its barycenter) and those due to the multi-well structure (see Cor. 1.2(iv) and Rem. 3.1). However, the homogenized density $\bar{g}$ in (4) is given by a complicated formula, and our paper can be seen as a first attempt in the scope of homogenization with gradient oscillations analysis.

Denote the set of all Young measures on $\Omega \times \mathbb{M}$ by $\mathcal{Y}(\Omega ; \mathbb{M})$. For each $\varepsilon>0$, let $\mathcal{F}_{\varepsilon}: \mathcal{Y}(\Omega ; \mathbb{M}) \rightarrow[0,+\infty]$ be defined by

$$
\mathcal{F}_{\varepsilon}(\mu):=\left\{\begin{array}{cl}
\int_{\Omega}\left(\int_{\mathbb{M}} f\left(\frac{x}{\varepsilon}, \xi\right) \mathrm{d} \mu_{x}(\xi)\right) \mathrm{d} x & \text { if } \mu=\mu_{x} \otimes \mathrm{d} x \in \Delta(\mathcal{U}) \\
+\infty & \text { otherwise }
\end{array}\right.
$$

where

$$
\Delta(\mathcal{U}):=\left\{\mu_{x} \otimes \mathrm{d} x \in \mathcal{Y}(\Omega ; \mathbb{M}): \mu_{x}=\delta_{\nabla u(x)} \text { with } u \in \mathcal{U}\right\}
$$

Let $\mathcal{P}(\mathbb{M})$ be the set of all probability measures on $\mathbb{M}$, and, for every $\xi \in \mathbb{M}$, let $\mathcal{H}_{\xi}(\mathbb{M})$ be the set of $\lambda \in \mathcal{P}(\mathbb{M})$ fulfilling the following three conditions ( $c f$. also Rem. 2.2):

$-\int_{\mathbb{M}} \zeta \mathrm{d} \lambda(\zeta)=\xi$

- $h(\xi) \leq \int_{\mathbb{M}} h(\zeta) \mathrm{d} \lambda(\zeta)$ for every quasiconvex function $h: \mathbb{M} \rightarrow \mathbb{R}$ bounded below and satisfying $h(\zeta) \leq$ $c\left(1+|\zeta|^{p}\right)$ for all $\zeta \in \mathbb{M}$ and some $c>0$;

$-\int_{\mathbb{M}}|\zeta|^{p} \mathrm{~d} \lambda(\zeta)<+\infty$.

For each bounded open set $A \subset \mathbb{R}^{N}$, we define $\mathfrak{S}_{A}(\xi, \cdot): \mathcal{H}_{\xi}(\mathbb{M}) \rightarrow[0,+\infty[$ by

$$
\mathfrak{S}_{A}(\xi, \lambda):=\inf \left\{\int_{A}\left(\int_{\mathbb{M}} f(x, \zeta) \mathrm{d} \sigma_{x}(\zeta)\right) \mathrm{d} x: \sigma_{x} \otimes \mathrm{d} x \in \nabla \mathcal{Y}_{\xi}(A), f_{A} \sigma_{x} \mathrm{~d} x=\lambda\right\}
$$

where $\nabla \mathcal{Y}_{\xi}(A)$ is the set of all $l_{\xi}+W_{0}^{1, p}\left(A ; \mathbb{R}^{m}\right)$-gradient Young measures, $l_{\xi}$ denoting the affine function with constant gradient $\xi$. The equality $f_{A} \sigma_{x} \mathrm{~d} x=\lambda$ means that $f_{A}\left(\int_{\mathbb{M}} \varphi(\zeta) \mathrm{d} \sigma_{x}(\zeta)\right) \mathrm{d} x=\langle\lambda, \varphi\rangle$ for all $\varphi$ in the space $C_{c}(\mathbb{M})$ of all real-valued and continuous functions with compact support on $\mathbb{M}$. Let $g: \mathbb{M} \times \mathcal{P}(\mathbb{M}) \rightarrow$ $[0,+\infty]$ be the measurable function defined by

$$
g(\xi, \lambda):=\left\{\begin{array}{cl}
\inf _{k \in \mathbb{N}^{*}} \frac{\mathfrak{S}_{k Y}(\xi, \lambda)}{k^{N}} & \text { if } \lambda \in \mathcal{H}_{\xi}(\mathbb{M}) \\
+\infty & \text { otherwise }
\end{array}\right.
$$

Then, for each fixed $\xi$ in $\mathbb{M}$, we consider $\bar{g}(\xi, \cdot): \mathcal{P}(\mathbb{M}) \rightarrow[0,+\infty]$, the weak lower semicontinuous envelope of $g(\xi, \cdot)$, i.e., the function defined by

$$
\bar{g}(\xi, \lambda):=\inf \left\{\varliminf_{n \rightarrow+\infty} g\left(\xi, \lambda_{n}\right): \mathcal{P}(\mathbb{M}) \ni \lambda_{n} \stackrel{*}{\rightarrow} \lambda\right\}
$$

where $\lambda_{n} \stackrel{*}{\rightarrow} \lambda$ means that for every $\varphi \in C_{c}(\mathbb{M}), \lim _{n \rightarrow+\infty}\left\langle\lambda_{n}, \varphi\right\rangle=\langle\lambda, \varphi\rangle$. It is worth noticing that, if $f$ does not depend on $x$, then $\bar{g}(\xi, \lambda)=\int_{\mathbb{M}} f(\zeta) \mathrm{d} \lambda(\zeta)$ for all $\xi \in \mathbb{M}$ and all $\lambda \in \mathcal{H}_{\xi}(\mathbb{M})$ (cf. Prop. 2.7). Let 
$\overline{\mathcal{F}}: \mathcal{Y}(\Omega ; \mathbb{M}) \rightarrow[0,+\infty]$ be defined by

$$
\overline{\mathcal{F}}(\mu):=\left\{\begin{array}{cl}
\int_{\Omega} \bar{g}\left(\operatorname{bar}\left(\mu_{x}\right), \mu_{x}\right) \mathrm{d} x & \text { if } \mu=\mu_{x} \otimes \mathrm{d} x \in \mathfrak{U} \\
+\infty & \text { otherwise }
\end{array}\right.
$$

with $\operatorname{bar}\left(\mu_{x}\right):=\int_{\mathbb{M}} \zeta \mathrm{d} \mu_{x}(\zeta)$. Here are the main results of the paper.

Theorem 1.1. Under $\left(\mathrm{C}_{1}\right)$ and $\left(\mathrm{C}_{2}\right)$, we have $\overline{\mathcal{F}}=\Gamma($ nar $)-\lim _{\varepsilon \rightarrow 0} \mathcal{F}_{\varepsilon}$.

The $\Gamma$-convergence process stated in Theorem 1.1 is taken with respect to the narrow convergence of Young measures because of its compactness property ( $c f$. Prokhorov's compactness theorem in Sect. 2 and Cor. 1.2(iii)).

According to the previous discussion, $\overline{\mathcal{F}}$ (resp. $\bar{g}$ ) in (5) (resp. (4)) can be called the homogenized freeenergy functional (resp. homogenized free-energy density) of the material in terms of Young measures. Since $F_{\varepsilon}(u)=\mathcal{F}_{\varepsilon}\left(\delta_{\nabla u(x)} \otimes \mathrm{d} x\right)$ for all $u \in \mathcal{U}$, as a direct consequence of Theorem 1.1, Proposition 2.3 and Remark 2.1, we obtain

Corollary 1.2. Under the hypotheses of Theorem 1.1, we have:

(i) $\lim _{\varepsilon \rightarrow 0} \inf _{\mathcal{U}} F_{\varepsilon}=\min _{\mathfrak{U}} \overline{\mathcal{F}}=\min _{\mathcal{U}} F_{\text {hom }}$;

(ii) if $\delta_{\nabla u_{\varepsilon}(x)} \otimes \mathrm{d} x \stackrel{\text { nar }}{\rightarrow} \mu$, with $\left\{u_{\varepsilon}\right\}_{\varepsilon}$ an $\varepsilon$-minimizing sequence for $F_{\varepsilon}$ in $\mathcal{U}$, then $\mu$ is a minimizer for $\overline{\mathcal{F}}$ in $\mathfrak{U}$

(iii) if $\mu$ is a minimizer for $\overline{\mathcal{F}}$ in $\mathfrak{U}$, then, there exists an $\varepsilon$-minimizing sequence $\left\{u_{\varepsilon}\right\}_{\varepsilon}$ for $F_{\varepsilon}$ in $\mathcal{U}$, such that $\delta_{\nabla u_{\varepsilon}(x)} \otimes \mathrm{d} x \stackrel{\text { nar }}{\rightarrow} \mu ;$

(iv) $u$ is a minimizer for $F_{\text {hom }}$ in $\mathcal{U}$ if and only if, there exists a minimizer $\mu=\mu_{x} \otimes \mathrm{d} x$ for $\overline{\mathcal{F}}$ in $\mathfrak{U}$, such that $\nabla u(x)=\operatorname{bar}\left(\mu_{x}\right)$ for a.e. $x \in \Omega$.

Remark 1.3. In contrast to the relaxed functional in (3), the homogenized functional in (5) is "quasi-local": it is local with respect to $\mathrm{d} x$ but, in general, non-local with respect to $\mu_{x}$, i.e., $\bar{g}$ has, a priori, no integral representation with respect to $\mu_{x}$.

Finally, the following result makes clear the link between $f_{\text {hom }}$ and $\bar{g}$.

Theorem 1.4. Assume that conditions $\left(\mathrm{C}_{1}\right)$ and $\left(\mathrm{C}_{2}\right)$ hold. Then,

$$
f_{\text {hom }}(\xi)=\inf \left\{\int_{Y} \bar{g}\left(\xi, \mu_{x}\right) \mathrm{d} x: \mu_{x} \otimes \mathrm{d} x \in \nabla \mathcal{Y}(Y), \operatorname{bar}\left(\mu_{x}\right)=\xi\right\}
$$

for all $\xi \in \mathbb{M}$, where $\nabla \mathcal{Y}(Y)$ denotes the set of all $W^{1, p}\left(Y ; \mathbb{R}^{m}\right)$-gradient Young measures.

A concrete example of our nonlinear homogenization process can be given when considering the free-energy functional of a polycristal with shape $\Omega$. In this particular case

$$
f(x, \xi)=h\left({ }^{t} R(x) \xi R(x)\right),
$$

where $R$ is a spatially periodic (or random) piecewise constant rotation-valued function, and $h$ has a finite number of wells (in the setting of linear elasticity these wells are called the stress-free strains of martensite variants, see Bhattacharya and Kohn [5] for more details). Minimizers of the homogenized free-energy functional $F_{\text {hom }}$ in (2) characterize the mixtures of martensite variants in the austenite/martensite phase transformation below the transition temperature. According to the point of view of Bhattacharya and Kohn, and taking formula (6) into account, we can say that $\bar{g}$ is the microscopic free-energy density corresponding to the macroscopic one $f_{\text {hom }}$.

The plan of the paper is as follows. Section 2 presents some preliminaries. In Section 2.1 we review some of the standard facts on Young (and gradient Young) measures. In Section 2.2, we briefly recall the notion of $\Gamma$-convergence. In Section 2.3 we point out a subadditive result ( $c f$. Prop. 2.4) for the set function $A \mapsto \mathfrak{S}_{A}(\xi, \lambda)$ 
with $\xi \in \mathbb{M}$ and $\lambda \in \mathcal{H}_{\xi}(\mathbb{M})$ (Prop. 2.4 is used in the proof of the upper bound in Th. 1.1). Properties of $\bar{g}$ (cf. Props. 2.5 and 2.7) are established in Section 2.4. Section 3 (resp Sect. 4) is devoted to the proof of Theorem 1.1 (resp. Th. 1.4). Finally, in Section 5 we discuss on some open questions and possible extensions.

\section{Preliminaries}

\subsection{Young measures}

Let $N, m \geq 1$ be two integers let $\mathbb{M}$ denote the space of all real $m \times N$ matrices, and consider a bounded open set $\Omega \subset \mathbb{R}^{N}$. A Young measure on $\Omega \times \mathbb{M}$ is a positive Radon measure $\mu$ on $\Omega \times \mathbb{M}$ such that $\mu(B \times \mathbb{M})=|B|$ for all Borel set $B \subset \Omega$, where $|B|$ denotes the Lebesgue measure of $B$. The set of all Young measures on $\Omega \times \mathbb{M}$ is denoted by $\mathcal{Y}(\Omega ; \mathbb{M})$. By $C \operatorname{th}^{b}(\Omega ; \mathbb{M})$ we denote the space of all bounded Carathéodory integrands on $\Omega \times \mathbb{M}$. Given $\mu \in \mathcal{Y}(\Omega ; \mathbb{M})$ and $\left\{\mu_{n}\right\}_{n \geq 1} \subset \mathcal{Y}(\Omega ; \mathbb{M})$, we say that $\mu_{n}$ narrow converges to $\mu$, and we write $\mu_{n} \stackrel{\text { nar }}{\longrightarrow} \mu$, if for every $\varphi \in C \operatorname{th}^{b}(\Omega ; \mathbb{M})$,

$$
\lim _{n \rightarrow+\infty} \int_{\Omega \times \mathbb{M}} \varphi(x, \xi) \mathrm{d} \mu_{n}(x, \xi)=\int_{\Omega \times \mathbb{M}} \varphi(x, \xi) \mathrm{d} \mu(x, \xi) .
$$

We denote the set of all probability measures on $\mathbb{M}$ by $\mathcal{P}(\mathbb{M})$. For a proof of the following theorem we refer to [13], Theorem 10, p. 14 (see also [22], Th. A4 and Cor. A5).

Slicing theorem. Given $\mu \in \mathcal{Y}(\Omega ; \mathbb{M})$, there exists a unique (up to the equality a.e.) family $\left\{\mu_{x}\right\}_{x \in \Omega} \subset \mathcal{P}(\mathbb{M})$ such that:

(i) the function $x \mapsto \int_{\Omega \times \mathbb{M}} \varphi(x, \xi) \mathrm{d} \mu_{x}$ is measurable;

(ii) $\int_{\Omega \times \mathbb{M}} \varphi(x, \xi) \mathrm{d} \mu(x, \xi)=\int_{\Omega}\left(\int_{\mathbb{M}} \varphi(x, \xi) \mathrm{d} \mu_{x}(\xi)\right) \mathrm{d} x$,

for every $\varphi \in L_{\mu}^{1}(\Omega \times \mathbb{M})$. To summarize it, we will write $\mu=\mu_{x} \otimes \mathrm{d} x$.

The slicing theorem leads to the following version of the narrow convergence, (see [22] for more details). Given $\mu \in \mathcal{Y}(\Omega ; \mathbb{M})$ and $\left\{\mu_{n}=\mu_{x}^{n} \otimes \mathrm{d} x\right\}_{n \geq 1} \subset \mathcal{Y}(\Omega ; \mathbb{M})$, we have: $\mu_{n} \stackrel{\text { nar }}{\longrightarrow} \mu$ if and only if for every $\psi \in C_{c}(\mathbb{M})$,

$$
\int_{\mathbb{M}} \psi(\xi) \mathrm{d} \mu_{x}^{n}(\xi) \rightarrow \int_{\mathbb{M}} \psi(\xi) \mathrm{d} \mu_{x}(\xi) \text { in } L^{\infty}(\Omega) \text { weak }^{*}
$$

i.e., for every $\psi \in C_{c}(\mathbb{M})$ and every $\varphi \in L^{1}(\Omega)$,

$$
\lim _{n \rightarrow+\infty} \int_{\Omega} \varphi(x)\left(\int_{\mathbb{M}} \psi(\xi) \mathrm{d} \mu_{x}^{n}(\xi)\right) \mathrm{d} x=\int_{\Omega} \varphi(x)\left(\int_{\mathbb{M}} \psi(\xi) \mathrm{d} \mu_{x}(\xi)\right) \mathrm{d} x
$$

We say that $\left\{\mu_{n}\right\} \subset \mathcal{Y}(\Omega ; \mathbb{M})$ is tight if for every $\delta>0$, there exists a compact set $K \subset \mathbb{M}$ such that $\sup \left\{\mu_{n}(\Omega \times\right.$ $(\mathbb{M} \backslash K)): n \geq 1\}<\delta$. A proof of the following compactness result can be found in [22], Theorem 11 (see also [23], Th. 7 and Comments 1), 2) and 3)).

Prokhorov's compactness theorem. If $\left\{\mu_{n}\right\} \subset \mathcal{Y}(\Omega ; \mathbb{M})$ is tight, then there exists $\mu \in \mathcal{Y}(\Omega ; \mathbb{M})$ such that, up to a subsequence, $\mu_{n} \stackrel{\text { nar }}{\longrightarrow} \mu$.

Remark 2.1. A straightforward consequence of Prokhorov's compactness theorem is the following: if $\left\{\xi_{n}\right\}_{n \geq 1}$ is a bounded sequence in $L^{1}(\Omega ; \mathbb{M})$, then the sequence $\left\{\delta_{\xi_{n}(x)} \otimes \mathrm{d} x\right\}_{n \geq 1}$ is narrow relatively compact. Indeed, by Markov's inequality, i.e., $\left|\left\{x \in \Omega:\left|\xi_{n}(x)\right| \geq c\right\}\right| \leq(1 / c) \int_{\Omega}\left|\xi_{n}(x)\right| \mathrm{d} x$ for any $c>0$ and any $n \geq 1$, it is obvious that $\left\{\delta_{\xi_{n}(x)} \otimes \mathrm{d} x\right\}_{n \geq 1}$ is tight.

The result below is usually referred as the continuity theorem. For a proof we refer to [23], Theorem 6 and Comments 1), 2), 3) and 4). 
Continuity theorem. Let $\left\{\xi_{n}\right\}_{n \geq 1}$ be a sequence of measurable functions from $\Omega$ to $\mathbb{M}$, let $\varphi: \Omega \times \mathbb{M} \rightarrow \mathbb{R}$ be a Carathéodory integrand, and let $\mu \in \mathcal{Y}(\Omega ; \mathbb{M})$. If $\delta_{\xi_{n}(x)} \otimes \mathrm{d} x \stackrel{\text { nar }}{\rightarrow} \mu$ and the sequence $\left\{\varphi\left(\cdot, \xi_{n}\right)\right\}_{n \geq 1}$ is uniformly integrable, then

$$
\lim _{n \rightarrow+\infty} \int_{\Omega} \varphi\left(x, \xi_{n}(x)\right) \mathrm{d} x=\int_{\Omega \times \mathbb{M}} \varphi(x, \xi) \mathrm{d} \mu(x, \xi) .
$$

Finally, we say that $\mu \in \mathcal{Y}(\Omega ; \mathbb{M})$ is a $\mathcal{U}$-gradient Young measure if there exists a bounded sequence $\left\{u_{n}\right\}_{n \geq 1}$ in $\mathcal{U}$ such that $\delta_{\nabla u_{n}(x)} \otimes \mathrm{d} x \stackrel{\text { nar }}{\sim} \mu$. The set of all $\mathcal{U}$-gradient Young measures is denoted by $\mathfrak{U}$. A characterization of $\mathfrak{U}$ was established by Kinderlehrer and Pedregal in [16], Theorem 1.1 (see also [20], Th. 8.14 p. 150).

Kinderlehrer-Pedregal's characterization theorem. $\mu \in \mathfrak{U}$ if and only if the following three conditions hold:

(i) there exists $u \in \mathcal{U}$ such that $\nabla u(x)=\int_{\mathbb{M}} \zeta \mathrm{d} \mu_{x}(\zeta)=$ : bar $\left(\mu_{x}\right)$ for a.e. $x \in \Omega$;

(ii) $h\left(\int_{\mathbb{M}} \zeta \mathrm{d} \mu_{x}(\zeta)\right) \leq \int_{\mathbb{M}} h(\zeta) \mathrm{d} \mu_{x}(\zeta)$ for a.e. $x \in \Omega$ and for every quasiconvex function $h: \mathbb{M} \rightarrow \mathbb{R}$ bounded below, and satisfying $h(\zeta) \leq c\left(1+|\zeta|^{p}\right)$ for all $\zeta \in \mathbb{M}$ and some $c>0$;

(iii) $\int_{\Omega}\left(\int_{\mathbb{M}}|\zeta|^{p} \mathrm{~d} \mu_{x}(\zeta)\right) \mathrm{d} x<+\infty$.

Remark 2.2. Given $\xi \in \mathbb{M}$ and a bounded open set $A \subset \mathbb{R}^{N}$, the set of all $l_{\xi}+W_{0}^{1, p}\left(A ; \mathbb{R}^{m}\right)$-gradient Young measures is denoted by $\nabla \mathcal{Y}_{\xi}(A)$. Similarly, we have: $\mu \in \nabla \mathcal{Y}_{\xi}(A)$ if and only if (i), (ii) and (iii) are satisfied with $\Omega$ replaced by $A$ and $\mathcal{U}$ by $l_{\xi}+W_{0}^{1, p}\left(A ; \mathbb{R}^{m}\right)$. Thus, the elements of $\mathcal{H}_{\xi}(\mathbb{M})$ are the homogeneous elements of $\nabla \mathcal{Y}_{\xi}(A)$ whose barycenter is equal to $\xi$, i.e.,

$$
\mathcal{H}_{\xi}(\mathbb{M}) \equiv\left\{\mu_{x} \otimes \mathrm{d} x \in \nabla \mathcal{Y}_{\xi}(A): \forall x \in \Omega, \mu_{x}=\lambda \text { with } \lambda \in \mathcal{P}(\mathbb{M}) \text { and } \operatorname{bar}(\lambda)=\xi\right\} .
$$

\section{2. $\Gamma$-convergence}

Let $\left\{\mathcal{F}_{\varepsilon}\right\}_{\varepsilon}$ be a sequence of functionals from $\mathcal{Y}(\Omega ; \mathbb{M})$ to $[0,+\infty]$ and let $\overline{\mathcal{F}}: \mathcal{Y}(\Omega ; \mathbb{M}) \rightarrow[0,+\infty]$. We say that $\mathcal{F}_{\varepsilon} \Gamma$ (nar)-converges to $\overline{\mathcal{F}}$ as $\varepsilon \rightarrow 0$, and we write $\overline{\mathcal{F}}=\Gamma\left(\right.$ nar)- $\lim _{\varepsilon \rightarrow 0} \mathcal{F}_{\varepsilon}$, if the following two assertions hold:

Lower bound: for every $\mu \in \mathcal{Y}(\Omega ; \mathbb{M})$, and every $\mu_{\varepsilon} \stackrel{\text { nar }}{\rightarrow} \mu$,

$$
\overline{\mathcal{F}}(\mu) \leq \varliminf_{\varepsilon \rightarrow 0} \mathcal{F}_{\varepsilon}\left(\mu_{\varepsilon}\right)
$$

Upper bound: for every $\mu \in \mathcal{Y}(\Omega ; \mathbb{M})$, there exists $\mu_{\varepsilon} \stackrel{\text { nar }}{\rightarrow} \mu$ such that

$$
\overline{\mathcal{F}}(\mu) \geq \varlimsup_{\varepsilon \rightarrow 0} \mathcal{F}_{\varepsilon}\left(\mu_{\varepsilon}\right)
$$

The following proposition is a well-known result that makes precise the variational nature of $\Gamma$-convergence.

Proposition 2.3. If $\overline{\mathcal{F}}=\Gamma($ nar $)-\lim _{\varepsilon \rightarrow 0} \mathcal{F}_{\varepsilon}$ and if $\left\{\mu_{\varepsilon}\right\}_{\varepsilon}$ is an $\varepsilon$-minimizing sequence for $\left\{\mathcal{F}_{\varepsilon}\right\}_{\varepsilon}$ which is narrow relatively compact, then any cluster point $\bar{\mu}$ of $\left\{\mu_{\varepsilon}\right\}_{\varepsilon}$ is a minimizer for $\overline{\mathcal{F}}$, and $\lim _{\varepsilon \rightarrow 0} \inf \mathcal{F}_{\varepsilon}\left(\mu_{\varepsilon}\right)=\overline{\mathcal{F}}(\bar{\mu})$.

For a proof and a deeper discussion of the $\Gamma$-convergence theory we refer the reader to the books $[3,7,11]$.

\subsection{A subadditive result}

Denote the class of all bounded open subsets of $\mathbb{R}^{N}$ by $\mathcal{O}_{b}$. A set function $\mathfrak{S}: \mathcal{O}_{b} \rightarrow\left[0,+\infty\left[, A \mapsto \mathfrak{S}_{A}\right.\right.$, is called subadditive if $\mathfrak{S}_{A} \leq \mathfrak{S}_{A^{\prime}}+\mathfrak{S}_{A^{\prime \prime}}$ for all $A, A^{\prime}, A^{\prime \prime} \in \mathcal{O}_{b}$ such that $A^{\prime} \subset A, A^{\prime \prime} \subset A$ and $\left|A^{\prime} \cap A^{\prime \prime}\right|=0$, $\left|A \backslash A^{\prime} \cup A^{\prime \prime}\right|=0$. The following well-known result is substantially the subadditive ergodic theorem of Akcoglu and Krengel (see [1]) in the deterministic case. For a proof we refer to [17], Theorem 2.1 (see also [2], Lem. B.1). 
Akcoglu-Krengel's subadditive theorem. Let $C u b\left(\mathbb{R}^{N}\right)$ be the class of all open cubes in $\mathbb{R}^{N}$, and consider a subadditive set function $\mathfrak{S}: \mathcal{O}_{b} \rightarrow[0,+\infty$ [ satisfying the following two conditions:

$\left(\mathrm{S}_{1}\right) \mathfrak{S}_{A} \leq c|A|$ for all $A \in \mathcal{O}_{b}$ and some $c>0$;

$\left(\mathrm{S}_{2}\right) \mathfrak{S}$ is $\mathbb{Z}^{N}$-invariant, i.e., $\mathfrak{S}_{A+z}=\mathfrak{S}_{A}$ for all $z \in \mathbb{Z}^{N}$ and all $A \in \mathcal{O}_{b}$.

Then, for every $Q \in C u b\left(\mathbb{R}^{N}\right)$ and every real sequence $\left\{r_{k}\right\}_{k \geq 1}$ with $r_{k} \rightarrow+\infty$ as $k \rightarrow+\infty$,

$$
\lim _{k \rightarrow+\infty} \frac{\mathfrak{S}_{r_{k} Q}}{\left|r_{k} Q\right|}=\inf _{k \in \mathbb{N}^{*}} \frac{\mathfrak{S}_{k Y}}{k^{N}}
$$

In our framework, we are led to consider, for each $\xi \in \mathbb{M}$ and each $\lambda \in \mathcal{P}(\mathbb{M})$, the set function $\mathcal{O}_{b} \ni A \mapsto \mathfrak{S}_{A}(\xi, \lambda)$ given by

$$
\mathfrak{S}_{A}(\xi, \lambda):=\inf \left\{\int_{A}\left(\int_{\mathbb{M}} f(x, \zeta) \mathrm{d} \sigma_{x}(\zeta)\right) \mathrm{d} x: \sigma_{x} \otimes \mathrm{d} x \in \Gamma_{A}(\xi, \lambda)\right\},
$$

where $\Gamma_{A}: \mathbb{M} \times \mathcal{P}(\mathbb{M}) \Longrightarrow \mathcal{Y}(A ; \mathbb{M})$ is the multifunction defined by

$$
\Gamma_{A}(\xi, \lambda):=\left\{\sigma_{x} \otimes \mathrm{d} x \in \nabla \mathcal{Y}_{\xi}(A): f_{A} \sigma_{x} \mathrm{~d} x=\lambda\right\}
$$

According to Remark 2.2 , it is clear that for every $A \in \mathcal{O}_{b}$,

$$
\Gamma_{A}(\xi, \lambda)=\emptyset \text { if and only if } \lambda \notin \mathcal{H}_{\xi}(\mathbb{M})
$$

For $\lambda \in \mathcal{H}_{\xi}(\mathbb{M})$ with $\xi \in \mathbb{M}$, we see that $\lambda \otimes \mathrm{d} x \in \Gamma_{A}(\xi, \lambda)$. From the second inequality in $\left(\mathrm{C}_{2}\right)$, it follows that $\mathfrak{S}_{A}(\xi, \lambda) \leq \beta\left(1+\int_{\mathbb{M}}|\zeta|^{p} \mathrm{~d} \lambda(\zeta)\right)|A|$ for all $A \in \mathcal{O}_{b}$. Thus $\mathfrak{S}_{(\cdot)}(\xi, \lambda)$ satisfies $\left(\mathrm{S}_{1}\right)$. Condition $\left(\mathrm{C}_{1}\right)$ makes it is obvious that $\left(\mathrm{S}_{2}\right)$ holds, and we let the reader to verify that $\mathfrak{S}_{(\cdot)}(\xi, \lambda)$ is subadditive. Applying AkcogluKrengel's subadditive theorem, we obtain the following proposition used in the proof of the upper bound in Theorem 1.1 ( $c f$. Sect. 3.2).

Proposition 2.4. If $\left(\mathrm{C}_{1}\right)$ and the second inequality in $\left(\mathrm{C}_{2}\right)$ hold, then for every $\xi \in \mathbb{M}$ and every $\lambda \in \mathcal{H}_{\xi}(\mathbb{M})$,

$$
\lim _{k \rightarrow+\infty} \frac{\mathfrak{S}_{k Y}(\xi, \lambda)}{k^{N}}=\inf _{k \in \mathbb{N}^{*}} \frac{\mathfrak{S}_{k Y}(\xi, \lambda)}{k^{N}} .
$$

\subsection{Properties of $\bar{g}$}

We begin with the following proposition.

Proposition 2.5. For every $\mu_{x} \otimes \mathrm{d} x \in \mathfrak{U}$, we have:

(i) the function $x \mapsto \bar{g}\left(\operatorname{bar}\left(\mu_{x}\right), \mu_{x}\right)$ is measurable;

(ii) if $\left(\mathrm{C}_{2}\right)$ holds then $\alpha \int_{\mathbb{M}}|\zeta|^{p} \mathrm{~d} \mu_{x}(\zeta) \leq \bar{g}\left(\operatorname{bar}\left(\mu_{x}\right), \mu_{x}\right) \leq \beta\left(1+\int_{\mathbb{M}}|\zeta|^{p} \mathrm{~d} \mu_{x}(\zeta)\right)$ for a.e. $x \in \Omega$.

Remark 2.6. As a consequence of Kinderlehrer-Pedregal's characterization theorem(iii) and the second inequality in Proposition 2.5(ii), we have $\operatorname{dom}(\overline{\mathcal{F}})=\mathfrak{U}$.

Proof of Proposition 2.5. (i) For every $A \in \mathcal{O}_{b}$, the mesurability of the function

$$
\mathbb{M} \times \mathcal{P}(\mathbb{M}) \ni(\xi, \lambda) \mapsto \mathfrak{S}_{A}(\xi, \lambda)=\inf \left\{\mathfrak{F}\left(\sigma_{x} \otimes \mathrm{d} x\right): \sigma_{x} \otimes \mathrm{d} x \in \Gamma_{A}(\xi, \lambda)\right\}
$$

where $\mathfrak{F}: \mathcal{Y}(A ; \mathbb{M}) \rightarrow[0,+\infty]$ is defined by $\mathfrak{F}\left(\sigma_{x} \otimes \mathrm{d} x\right)=\int_{A}\left(\int_{\mathbb{M}} f(x, \zeta) \mathrm{d} \sigma_{x}(\zeta)\right) \mathrm{d} x$ and $\Gamma_{A}: \mathbb{M} \times \mathcal{P}(\mathbb{M}) \Longrightarrow \mathcal{Y}(A ; \mathbb{M})$ is given by (7), comes from [9], Lemma III.39. Taking (8) into account, we see that $g(\xi, \lambda)=\inf _{k \in \mathbb{N}^{*}} \mathfrak{S}_{k Y}(\xi, \lambda) / k^{N}$ for all $\xi \in \mathbb{M}$ and all $\lambda \in \mathcal{P}(\mathbb{M})$, hence $g$ is measurable and (i) follows. 
(ii) Fix $x \in \Omega$. From the second inequality in $\left(\mathrm{C}_{2}\right)$, we have

$$
\int_{Y}\left(\int_{\mathbb{M}} f(y, \zeta) \mathrm{d} \mu_{x}(\zeta)\right) \mathrm{d} y \leq \beta\left(1+\int_{\mathbb{M}}|\zeta|^{p} \mathrm{~d} \mu_{x}(\zeta)\right)
$$

and the second inequality in (ii) follows since $\bar{g}\left(\operatorname{bar}\left(\mu_{x}\right), \mu_{x}\right) \leq \mathfrak{S}_{Y}\left(\operatorname{bar}\left(\mu_{x}\right), \mu_{x}\right)$ and $\mu_{x} \otimes \mathrm{d} y \in \Gamma_{Y}\left(\operatorname{bar}\left(\mu_{x}\right), \mu_{x}\right)$. On the other hand, considering $\left\{\lambda_{n}\right\}_{n \geq 1} \subset \mathcal{P}(\mathbb{M})$ such that $\lambda_{n} \stackrel{*}{\rightarrow} \mu_{x}$ and $\bar{g}\left(\operatorname{bar}\left(\mu_{x}\right), \mu_{x}\right)=\lim _{n \rightarrow+\infty} g\left(\operatorname{bar}\left(\mu_{x}\right)\right.$, $\left.\lambda_{n}\right)$, and using the first inequality in $\left(\mathrm{C}_{2}\right)$, we see that

$$
\bar{g}\left(\operatorname{bar}\left(\mu_{x}\right), \mu_{x}\right) \geq \underset{n \rightarrow+\infty}{\lim _{\rightarrow}} \alpha \int_{\mathbb{M}}|\zeta|^{p} \mathrm{~d} \lambda_{n}(\zeta) \geq \alpha \int_{\mathbb{M}}|\zeta|^{p} \mathrm{~d} \mu_{x}(\zeta)
$$

which completes the proof.

The next proposition shows that the relaxation theorem of Kinderlehrer and Pedregal is a particular case of Corollary 1.2.

Proposition 2.7. If the second inequality in $\left(\mathrm{C}_{2}\right)$ holds and if $f$ does not depend on $x$, then $\bar{g}(\xi, \lambda)=$ $\int_{\mathbb{M}} f(\zeta) \mathrm{d} \lambda(\zeta)$ for all $\xi \in \mathbb{M}$ and all $\lambda \in \mathcal{H}_{\xi}(\mathbb{M})$.

Proof. Taking the second inequality in $\left(\mathrm{C}_{2}\right)$ into account, it is clear that for every $k \geq 1$, every $\xi \in \mathbb{M}$ and every $\lambda \in \mathcal{H}_{\xi}(\mathbb{M}), f_{k Y}\left(\int_{\mathbb{M}} f(\zeta) \mathrm{d} \sigma_{x}(\zeta)\right) \mathrm{d} x=\int_{\mathbb{M}} f(\zeta) \mathrm{d} \lambda(\zeta)$ whenever $\sigma_{x} \otimes \mathrm{d} x \in \nabla \mathcal{Y}_{\xi}(k Y)$ with $f_{k y} \sigma_{x} \mathrm{~d} x=\lambda$. It follows that $g(\xi, \lambda)=\int_{\mathbb{M}} f(\zeta) \mathrm{d} \lambda(\zeta)$ for all $\xi \in \mathbb{M}$ and all $\lambda \in \mathcal{H}_{\xi}(\mathbb{M})$, which gives the desired conclusion because the mapping $\lambda \mapsto \int_{\mathbb{M}} f(\zeta) \mathrm{d} \lambda(\zeta)$ is weakly lower semicontinuous on $\mathcal{P}(\mathbb{M})$.

\section{Proof of Theorem 1.1}

\subsection{Proof of the lower bound}

Let $\mu=\mu_{x} \otimes \mathrm{d} x \in \mathcal{Y}\left(\Omega ; \mathbb{R}^{m}\right)$, and $\mu_{\varepsilon} \stackrel{\text { nar }}{\rightarrow} \mu$. We have to prove that

$$
\overline{\mathcal{F}}(\mu) \leq \varliminf_{\varepsilon \rightarrow 0} \mathcal{F}_{\varepsilon}\left(\mu_{\varepsilon}\right)
$$

Without loss of generality we can assume that

$$
\varliminf_{\varepsilon \rightarrow 0} \mathcal{F}_{\varepsilon}\left(\mu_{\varepsilon}\right)<+\infty
$$

Thus, $\mu_{\varepsilon} \in \Delta(\mathcal{U})$, i.e., there exists $u_{\varepsilon} \in \mathcal{U}$ such that $\mu_{\varepsilon}=\delta_{\nabla u_{\varepsilon}(x)} \otimes \mathrm{d} x$, and so

$$
\mathcal{F}_{\varepsilon}\left(\mu_{\varepsilon}\right)=\int_{\Omega} f\left(\frac{x}{\varepsilon}, \nabla u_{\varepsilon}(x)\right) \mathrm{d} x .
$$

From the first inequality in $\left(\mathrm{C}_{2}\right)$, we see that $\left\{\mu_{\varepsilon}\right\}_{\varepsilon}$ is tight. Using Prokorov's compactness theorem, we deduce that there exists $\bar{\mu} \in \mathfrak{U}$ such that (up to a subsequence) $\mu_{\varepsilon} \stackrel{\text { nar }}{\mu} \bar{\mu}(c f$. Rem. 2.1). Then $\mu=\bar{\mu}$, and so $\mu \in \mathfrak{U}$. According to Kinderlehrer-Pedregal's characterization theorem, there exists $u \in \mathcal{U}$ such that $\operatorname{bar}\left(\mu_{x}\right)=\nabla u(x)$ for a.e. $x \in \Omega$.

In order to obtain (9) we proceed in three steps. Firstly, using a standard blow-up technique near $x_{0}$, we show that is sufficient to prove

$$
\bar{g}\left(\nabla u\left(x_{0}\right), \mu_{x_{0}}\right) \leq \lim _{\rho \rightarrow 0} \lim _{\varepsilon \rightarrow 0} f_{\mathcal{Q}_{\rho}\left(x_{0}\right)} f\left(\frac{x}{\varepsilon}, \nabla u_{\varepsilon}(x)\right) \mathrm{d} x .
$$


The two last steps consist in establishing (11) by means of De Giorgi's slicing method together with a lower semicontinuous regularization.

Step 1 (localization and blow-up). Denote the space of all Radon measures on $\Omega$ by $\mathcal{M}(\Omega)$, and set $\mathcal{M}^{+}(\Omega):=$ $\{\Theta \in \mathcal{M}(\Omega): \Theta \geq 0\}$. Let $\left\{\Theta_{\varepsilon}\right\}_{\varepsilon} \subset \mathcal{M}^{+}(\Omega)$ be defined by

$$
\Theta_{\varepsilon}:=f\left(\frac{\dot{\varepsilon}}{\varepsilon}, \nabla u_{\varepsilon}\right) \mathrm{d} x
$$

By (10), $\left\{\Theta_{\varepsilon}\right\}_{\varepsilon}$ is bounded in $\mathcal{M}^{+}(\Omega)$, hence there exists $\Theta \in \mathcal{M}^{+}(\Omega)$ such that (up to a subsequence) $\Theta_{\varepsilon} \stackrel{*}{\rightarrow} \Theta$. As $\Theta(\Omega) \leq \underline{\lim }_{\varepsilon \rightarrow 0} \Theta_{\varepsilon}(\Omega)$, if we prove that

$$
\int_{\Omega} \bar{g}\left(\nabla u(x), \mu_{x}\right) \mathrm{d} x \leq \Theta(\Omega)
$$

then (9) will follow. Consider the Lebesgue decomposition of $\Theta=\Theta^{a}+\Theta^{s}$, where $\Theta^{a}, \Theta^{s} \in \mathcal{M}^{+}(\Omega)$ are respectively the absolutely continuous and the singular part with respect to $\mathrm{d} x$. Radon-Nikodym's theorem asserts that there exists $\theta \in L^{1}\left(\Omega ; \mathbb{R}^{+}\right)$such that $\Theta^{a}=\theta \mathrm{d} x$, and by Lebesgue's differentiation theorem,

$$
\theta\left(x_{0}\right)=\lim _{\rho \rightarrow 0} \frac{\Theta^{a}\left(\mathcal{Q}_{\rho}\left(x_{0}\right)\right)}{\left|\mathcal{Q}_{\rho}\left(x_{0}\right)\right|}=\lim _{\rho \rightarrow 0} \frac{\Theta\left(\mathcal{Q}_{\rho}\left(x_{0}\right)\right)}{\left|\mathcal{Q}_{\rho}\left(x_{0}\right)\right|}
$$

for a.e. $x_{0} \in \Omega$, where $\mathcal{Q}_{\rho}\left(x_{0}\right)$ is the open cube centered at $x_{0}$ and of side $\rho$. From now on, one fix any $x_{0}$ outside a negligible set of $\Omega$, such that the following four assertions hold:

$-\operatorname{bar}\left(\mu_{x_{0}}\right)=\nabla u\left(x_{0}\right)$;

- (12) holds;

- for every $\psi \in \mathcal{D}$,

$$
\lim _{\rho \rightarrow 0} f_{\mathcal{Q}_{\rho}\left(x_{0}\right)}\left(\int_{\mathbb{M}} \psi(\xi) \mathrm{d} \mu_{x}(\xi)\right) \mathrm{d} x=\int_{\mathbb{M}} \psi(\xi) \mathrm{d} \mu_{x_{0}}(\xi),
$$

where $\mathcal{D}$ is a countable subset of Lipschitz function from $\mathbb{M}$ to $\mathbb{R}$ which is dense in $C_{c}(\mathbb{M})$;

- for $\bar{u}: \mathbb{R}^{N} \rightarrow \mathbb{R}^{m}$ denoting the affine function defined by $\bar{u}(x):=u\left(x_{0}\right)+\nabla u\left(x_{0}\right) \cdot\left(x-x_{0}\right)$, we have (see [24], Th. 3.4.2)

$$
\lim _{\rho \rightarrow 0} \frac{1}{\rho} f_{\mathcal{Q}_{\rho}\left(x_{0}\right)}|u-\bar{u}| \mathrm{d} x=0 .
$$

As $\Theta_{\varepsilon} \stackrel{*}{\rightarrow} \Theta$, one has $\Theta\left(\mathcal{Q}_{\rho}\left(x_{0}\right)\right)=\lim _{\varepsilon \rightarrow 0} \Theta_{\varepsilon}\left(\mathcal{Q}_{\rho}\left(x_{0}\right)\right)$ whenever $\Theta\left(\partial \mathcal{Q}_{\rho}\left(x_{0}\right)\right)=0$. Since $\Theta$ is finite, $\Theta\left(\partial \mathcal{Q}_{\rho}\left(x_{0}\right)\right)=0$ for all but countably many $\rho>0$. In the sequel, we will take $\rho$ such that $\Theta\left(\partial \mathcal{Q}_{\rho}\left(x_{0}\right)\right)=0$. Consequently, it is sufficient to prove (11).

Step 2 (decreasing the energy by slicing De Giorgi's method). Fix any $t \in] 0,1\left[\right.$ and any $\ell \in \mathbb{N}^{*}$. For each $i \in\{0, \cdots, \ell\}$, define $\mathcal{Q}_{i}:=\mathcal{Q}_{t \rho+i(1-t) \rho / \ell}\left(x_{0}\right)$ and consider a cut-off function $\phi_{i}$ between $\mathcal{Q}_{i-1}$ and $\mathcal{Q}_{i}(i \geq 1)$ such that $\left\|\nabla \phi_{i}\right\|_{\infty} \leq \frac{2 \ell}{(1-t) \rho}$. Setting $u_{\varepsilon}^{i}(x):=\bar{u}(x)+\phi_{i}(x)\left(u_{\varepsilon}(x)-\bar{u}(x)\right)$, we have $u_{\varepsilon}^{i} \in l_{\nabla u\left(x_{0}\right)}+W^{1, p}\left(\mathcal{Q}_{\rho}\left(x_{0}\right) ; \mathbb{R}^{m}\right)$ and

$$
\nabla u_{\varepsilon}^{i}= \begin{cases}\nabla u_{\varepsilon} & \text { on } \mathcal{Q}_{i-1} \\ \nabla u\left(x_{0}\right)+\left(u_{\varepsilon}-\bar{u}\right) \otimes \nabla \phi_{i}+\phi_{i}\left(\nabla u_{\varepsilon}-\nabla u\left(x_{0}\right)\right) & \text { on } \mathcal{Q}_{i} \backslash \mathcal{Q}_{i-1} \\ \nabla u\left(x_{0}\right) & \text { on } \mathcal{Q}_{\rho}\left(x_{0}\right) \backslash \mathcal{Q}_{i} .\end{cases}
$$

Using the second inequality in $\left(\mathrm{C}_{2}\right)$, we obtain

$$
\frac{1}{\ell} \sum_{i=1}^{\ell} f_{\mathcal{Q}_{\rho}\left(x_{0}\right)} f\left(\frac{x}{\varepsilon}, \nabla u_{\varepsilon}^{i}(x)\right) \mathrm{d} x \leq f_{\mathcal{Q}_{\rho}\left(x_{0}\right)} f\left(\frac{x}{\varepsilon}, \nabla u_{\varepsilon}(x)\right) \mathrm{d} x+\hat{\mathrm{A}}(\varepsilon, \rho)
$$


with $\hat{\mathrm{A}}(\varepsilon, \rho):=\frac{1}{\ell} \sum_{i=1}^{\ell} \mathrm{A}_{i}(\varepsilon, \rho)$ and

$$
\mathrm{A}_{i}(\varepsilon, \rho):=\frac{1}{\rho^{N}} \int_{\mathcal{Q}_{i} \backslash \mathcal{Q}_{i-1}} f\left(\frac{x}{\varepsilon}, \nabla u_{\varepsilon}^{i}(x)\right) \mathrm{d} x+\beta\left(1+\left|\nabla u\left(x_{0}\right)\right|^{p}\right)\left(1-t^{N}\right) .
$$

Let $k_{\varepsilon} \in \mathbb{N}^{*}$ be the smallest integer such that $\frac{1}{\varepsilon} \mathcal{Q}_{\rho}\left(x_{0}\right) \subset k_{\varepsilon} Y+z_{\varepsilon}$ for an appropriate $z_{\varepsilon} \in \mathbb{Z}^{N}$. Consider $\bar{\imath}(\varepsilon, \rho, t, \ell)=: \bar{\imath} \in\{1, \cdots, \ell\}$ such that

$$
f_{\mathcal{Q}_{\rho}\left(x_{0}\right)} f\left(\frac{x}{\varepsilon}, \nabla u_{\varepsilon}^{\bar{\imath}}(x)\right) \mathrm{d} x \leq \frac{1}{\ell} \sum_{i=1}^{\ell} f_{\mathcal{Q}_{\rho}\left(x_{0}\right)} f\left(\frac{x}{\varepsilon}, \nabla u_{\varepsilon}^{i}(x)\right) \mathrm{d} x
$$

and define $w_{\varepsilon}^{\bar{\imath}} \in l_{\nabla u\left(x_{0}\right)}+W_{0}^{1, p}\left(k_{\varepsilon} Y ; \mathbb{R}^{m}\right)$ by

$$
w_{\varepsilon}^{\bar{\imath}}(x):= \begin{cases}\frac{1}{\varepsilon} v_{n}^{\bar{\tau}}\left(\varepsilon\left(x+z_{\varepsilon}\right)\right) & \text { if } x \in \frac{1}{\varepsilon} \mathcal{Q}_{\rho}\left(x_{0}\right)-z_{\varepsilon} \\ l_{\nabla u\left(x_{0}\right)}(x) & \text { if } x \in k_{\varepsilon} Y \backslash\left(\frac{1}{\varepsilon} \mathcal{Q}_{\rho}\left(x_{0}\right)-z_{\varepsilon}\right)\end{cases}
$$

with $v_{\varepsilon}^{\bar{\imath}} \in l_{\nabla u\left(x_{0}\right)}+W_{0}^{1, p}\left(\mathcal{Q}_{\rho}\left(x_{0}\right) ; \mathbb{R}^{m}\right)$ given by $v_{\varepsilon}^{\bar{\imath}}(x):=u_{\varepsilon}^{\bar{\imath}}(x)-u\left(x_{0}\right)+\nabla u\left(x_{0}\right) \cdot x_{0}$. By $\left(\mathrm{C}_{1}\right)$, we thus have

$$
f_{\frac{1}{\varepsilon} \mathcal{Q}_{\rho}\left(x_{0}\right)} f\left(x, \nabla w_{\varepsilon}^{\bar{\imath}}\left(x-z_{\varepsilon}\right)\right) \mathrm{d} x=f_{\mathcal{Q}_{\rho}\left(x_{0}\right)} f\left(\frac{x}{\varepsilon}, \nabla u_{\varepsilon}^{\bar{\imath}}(x)\right) \mathrm{d} x .
$$

Setting $\gamma:=\beta\left(1+\left|\nabla u\left(x_{0}\right)\right|\right)$ and $\Delta_{\varepsilon}:=k_{\varepsilon}^{-N}\left[k_{\varepsilon}^{N}-\left(k_{\varepsilon}-2\right)^{N}\right]$, it is easily seen that

$$
f_{k_{\varepsilon} Y} f\left(x, \nabla w_{\varepsilon}^{\bar{\imath}}(x)\right) \mathrm{d} x \leq f_{\frac{1}{\varepsilon} \mathcal{Q}_{\rho}\left(x_{0}\right)} f\left(x, \nabla w_{\varepsilon}^{\bar{\imath}}\left(x-z_{\varepsilon}\right)\right) \mathrm{d} x+\gamma \Delta_{\varepsilon} .
$$

Let $\lambda_{\varepsilon}^{\bar{\imath}} \in \mathcal{H}_{\nabla u\left(x_{0}\right)}(\mathbb{M})$ be defined by

$$
\lambda_{\varepsilon}^{\bar{\imath}}:=f_{k_{\varepsilon} Y} \delta_{\nabla w_{\varepsilon}^{\bar{i}}(x)} \mathrm{d} x .
$$

By definition, $\lim _{\varepsilon \rightarrow 0} k_{\varepsilon}=+\infty$, hence $\lim _{\varepsilon \rightarrow 0} \Delta_{\varepsilon}=0$, and consequently

$$
\begin{aligned}
\varlimsup_{\varepsilon \rightarrow 0} \inf _{k \in \mathbb{N}^{*}} \frac{\mathfrak{S}_{k Y}\left(\xi, \lambda_{\varepsilon}^{\bar{i}}\right)}{k^{N}} & =\varlimsup_{\varepsilon \rightarrow 0} g\left(\nabla u\left(x_{0}\right), \lambda_{\varepsilon}^{\bar{i}}\right) \\
& \leq \varlimsup_{n \rightarrow+\infty} f_{\frac{1}{\varepsilon} \mathcal{Q}_{\rho}\left(x_{0}\right)} f\left(x, \nabla w_{n}^{\bar{\imath}}\left(x-z_{\varepsilon}\right)\right) \mathrm{d} x .
\end{aligned}
$$

Using (14), we see that $\varlimsup_{\rho \rightarrow 0} \varlimsup_{\varepsilon \rightarrow 0} \hat{\mathrm{A}}(\varepsilon, \rho) \leq c\left[\left(1-t^{N}\right)+\frac{1}{\ell}\right]$, where $c>0$ is a constant independent of $\varepsilon$, $\rho, t$ and $\ell$. Taking (16), (17) and (18) into account, from (19) we deduce that

$$
\varlimsup_{\ell \rightarrow+\infty} \varlimsup_{t \rightarrow 1} \varlimsup_{\rho \rightarrow 0} \varlimsup_{\varepsilon \rightarrow 0} g\left(\nabla u\left(x_{0}\right), \lambda_{\varepsilon}^{\bar{\imath}}\right) \leq \lim _{\rho \rightarrow 0} \lim _{\varepsilon \rightarrow 0} f_{\mathcal{Q}_{\rho}\left(x_{0}\right)} f\left(\frac{x}{\varepsilon}, \nabla u_{\varepsilon}(x)\right) \mathrm{d} x .
$$

Step 3 (end of the proof). There is no loss of generality in assuming that there exist $\lambda_{\rho}(t, \ell), \lambda_{t}(\ell), \lambda_{\ell}, \lambda \in \mathcal{P}(\mathbb{M})$ such that $\lambda_{\varepsilon}^{\bar{\imath}} \stackrel{*}{\rightarrow} \lambda_{\rho}(t, \ell)$ as $\varepsilon \rightarrow 0, \lambda_{\rho}(t, \ell) \stackrel{*}{\rightarrow} \lambda_{t}(\ell)$ as $\rho \rightarrow 0, \lambda_{t}(\ell) \stackrel{*}{\rightarrow} \lambda_{\ell}$ as $t \rightarrow 1$ and $\lambda_{\ell} \stackrel{*}{\rightarrow} \lambda$ as $\ell \rightarrow+\infty$. We claim that

$$
\lambda=\mu_{x_{0}} .
$$


Indeed, given any $\varphi \in C_{c}(\mathbb{M})$ and any $\eta>0$, consider $\psi \in \mathcal{D}$ a $C$-Lipschitz function such that $\|\varphi-\psi\|_{\infty} \leq \eta$. Then,

Moreover,

$$
\left|\left\langle\lambda_{\varepsilon}^{\bar{\imath}}-\mu_{x_{0}}, \varphi\right\rangle\right| \leq\left|\left\langle\lambda_{\varepsilon}^{\bar{i}}-\mu_{x_{0}}, \psi\right\rangle\right|+2 \eta
$$

$$
\left|\left\langle\lambda_{\varepsilon}^{\bar{\imath}}-\mu_{x_{0}}, \psi\right\rangle\right| \leq\left|f_{\mathcal{Q}_{\rho}\left(x_{0}\right)} \psi\left(\nabla u_{\varepsilon}(x)\right) \mathrm{d} x-\left\langle\mu_{x_{0}}, \psi\right\rangle\right|+\left|\psi\left(\nabla u\left(x_{0}\right)\right)\right| \Delta_{\varepsilon}+\mathrm{B}_{\bar{\imath}}(\varepsilon, \rho)
$$

with

$$
\mathrm{B}_{\bar{\imath}}(\varepsilon, \rho):=f_{\mathcal{Q}_{\rho}\left(x_{0}\right)}\left|\psi\left(\nabla u_{\varepsilon}^{\bar{\imath}}(x)\right)-\psi\left(\nabla u_{\varepsilon}(x)\right)\right| \mathrm{d} x .
$$

Since $\mu_{\varepsilon} \stackrel{\text { nar }}{\longrightarrow} \mu$, from (13) we have

$$
\lim _{\rho \rightarrow 0} \lim _{\varepsilon \rightarrow 0}\left|f_{\mathcal{Q}_{\rho}\left(x_{0}\right)} \psi\left(\nabla u_{\varepsilon}(x)\right) \mathrm{d} x-\left\langle\mu_{x_{0}}, \psi\right\rangle\right|=0 .
$$

As $\psi$ is $C$-Lipschitz, using $\left(\mathrm{C}_{2}\right)$ and (15), we obtain the following estimate:

$$
\mathrm{B}_{\bar{\imath}}(\varepsilon, \rho) \leq \hat{C}\left(1-t^{N}\right)^{p-1 / p}\left[1+\left(\frac{\Theta_{\varepsilon}\left(\mathcal{Q}_{\rho}\left(x_{0}\right)\right)}{\left|\mathcal{Q}_{\rho}\left(x_{0}\right)\right|}\right)^{1 / p}\right]+\frac{2 C \ell}{(1-t) \rho} f_{\mathcal{Q}_{\rho}\left(x_{0}\right)}|u-\bar{u}| \mathrm{d} x
$$

with $\hat{C}:=\max \left\{C\left|\nabla u\left(x_{0}\right)\right|,(1 / \alpha)^{1 / p}\right\}$. Taking (14) into account, we see that

$$
\varlimsup_{\rho \rightarrow 0} \varlimsup_{\varepsilon \rightarrow 0} \mathrm{~B}_{\bar{\imath}}(\varepsilon, \rho) \leq \hat{C}\left(1-t^{N}\right)^{p-1 / p}\left(\left|\nabla u\left(x_{0}\right)\right|+\theta\left(x_{0}\right)^{1 / p}\right)
$$

with $\theta\left(x_{0}\right)$ given by (12). Thus $\lim _{\ell \rightarrow+\infty} \lim _{t \rightarrow 1} \varlimsup_{\rho \rightarrow 0} \overline{\lim }_{\varepsilon \rightarrow 0}\left|\left\langle\lambda_{\varepsilon}^{\bar{\imath}}-\mu_{x_{0}}, \psi\right\rangle\right|=0$. As $\eta$ is arbitrary, we deduce that for every $\varphi \in C_{c}(\mathbb{M})$,

and the claim follows. We thus have:

$$
\lim _{\ell \rightarrow+\infty} \lim _{t \rightarrow 1} \varlimsup_{\rho \rightarrow 0} \varlimsup_{\varepsilon \rightarrow 0}\left\langle\lambda_{\varepsilon}^{\bar{i}}, \varphi\right\rangle=\left\langle\mu_{x_{0}}, \varphi\right\rangle,
$$

$$
\begin{aligned}
& -\bar{g}\left(\nabla u\left(x_{0}\right), \lambda_{\rho}(t, \ell)\right) \leq \varliminf_{\varepsilon \rightarrow 0} g\left(\nabla u\left(x_{0}\right), \lambda_{\varepsilon}^{\bar{\imath}}\right) ; \\
& \left.-\bar{g}\left(\nabla u\left(x_{0}\right), \lambda_{t}(\ell)\right)\right) \leq \varliminf_{\rho \rightarrow 0} \bar{g}\left(\nabla u\left(x_{0}\right), \lambda_{\rho}(t, \ell)\right) ; \\
& -\bar{g}\left(\nabla u\left(x_{0}\right), \lambda_{\ell}\right) \leq \varliminf_{t \rightarrow 1} \bar{g}\left(\nabla u\left(x_{0}\right), \lambda_{t}(\ell)\right) ; \\
& -\bar{g}\left(\nabla u\left(x_{0}\right), \mu_{x_{0}}\right) \leq \varliminf_{\ell \rightarrow+\infty} \bar{g}\left(\nabla u\left(x_{0}\right), \lambda_{\ell}\right) .
\end{aligned}
$$

Hence, $\bar{g}\left(\mu_{x_{0}}, \nabla u\left(x_{0}\right)\right) \leq \varliminf_{\ell \rightarrow+\infty} \varliminf_{t \rightarrow 1} \varliminf_{\rho \rightarrow 0} \varliminf_{\varepsilon \rightarrow 0} g\left(\lambda_{\varepsilon}^{\bar{\imath}}, \nabla u\left(x_{0}\right)\right)$, and (11) follows from (20).

\subsection{Proof of the upper bound}

Let $\mu \in \mathcal{Y}(\Omega ; \mathbb{M})$. We have to prove that there exists $\left\{\mu_{\varepsilon}\right\}_{\varepsilon} \subset \mathcal{Y}(\Omega ; \mathbb{M})$ such that $\mu_{\varepsilon} \stackrel{\text { nar }}{\rightarrow} \mu$ and

$$
\overline{\mathcal{F}}(\mu) \geq \varlimsup_{\varepsilon \rightarrow 0} \mathcal{F}_{\varepsilon}\left(\mu_{\varepsilon}\right)
$$

Without loss of generality we can assume that $\overline{\mathcal{F}}(\mu)<+\infty$. Thus $\mu \in \mathfrak{U}$ (cf. Rem. 2.6), and

$$
\overline{\mathcal{F}}(\mu)=\int_{\Omega} \bar{g}\left(\operatorname{bar}\left(\mu_{x}\right), \mu_{x}\right) \mathrm{d} x .
$$

We proceed in three steps. For a comprehensive reading we refer to Remark 3.1 before Step 3. 
Step 1 (localization by generalized Riemann summation). Taking Proposition 2.5(i) into account and using [16], Lemma 5.1, we can assert that for every integer $j \geq 1$, there exists a countable family $\left(a_{i, j}+s_{i, j} \Omega\right)_{i \geq 1}$ of disjoint subsets of $\Omega$, with $a_{i, j} \in \Omega$ and $0<s_{i, j}<\frac{1}{j}$, such that: $\left|\Omega \backslash \cup_{i=1}^{\infty}\left(a_{i, j}+s_{i, j} \Omega\right)\right|=0$;

$$
\int_{\Omega} \bar{g}\left(\operatorname{bar}\left(\mu_{x}\right), \mu_{x}\right) \mathrm{d} x=\lim _{j \rightarrow+\infty} \sum_{i=1}^{\infty}\left|\Omega_{i, j}\right| \bar{g}\left(\operatorname{bar}\left(\mu_{a_{i, j}}\right), \mu_{a_{i, j}}\right)
$$

with $\Omega_{i, j}:=a_{i, j}+s_{i, j} \Omega ;$ and

$$
\int_{\Omega} \varphi(x)\left(\int_{\mathbb{M}} \psi(\xi) \mathrm{d} \mu_{x}(\xi)\right) \mathrm{d} x=\lim _{j \rightarrow+\infty} \sum_{i=1}^{\infty} \int_{\mathbb{M}} \psi(\xi) \mathrm{d} \mu_{a_{i, j}}(\xi) \int_{\Omega_{i, j}} \varphi(x) \mathrm{d} x
$$

for all $\varphi \in L^{1}(\Omega)$ and all $\psi \in \mathcal{D}$, where $\mathcal{D}$ is a dense countable subset of $C_{c}(\mathbb{M})$.

Step 2 (Proof of the upper bound on $\Omega_{i, j}$ ). Fix any $i, j \geq 1$. Set $\xi_{i, j}:=\operatorname{bar}\left(\mu_{a_{i, j}}\right)$ and consider $\left\{\lambda_{n}\right\}_{n \geq 1} \subset$ $\mathcal{H}_{\xi_{i, j}}(\mathbb{M})$ such that:

$\left(\mathrm{A}_{1}\right) \lambda_{n} \stackrel{*}{\rightarrow} \mu_{a_{i, j}}$, and so $\lambda_{n} \otimes \mathrm{d} x \stackrel{\text { nar }}{\rightarrow} \mu_{a_{i, j}} \otimes \mathrm{d} x$ as $n \rightarrow+\infty$;

$\left(\mathrm{B}_{1}\right) \lim _{n \rightarrow+\infty} g\left(\xi_{i, j}, \lambda_{n}\right)=\bar{g}\left(\xi_{i, j}, \mu_{a_{i, j}}\right)$.

By Proposition 2.4, we have $g\left(\xi_{i, j}, \lambda_{n}\right)=\lim _{k \rightarrow+\infty} \mathfrak{S}_{k Y}\left(\xi_{i, j}, \lambda_{n}\right) / k^{N}$ for all $n \geq 1$. Moreover, there is no loss of generality in assuming that to every $n, k \geq 1$, there corresponds $\sigma_{x}^{n, k} \otimes \mathrm{d} x \in \nabla \mathcal{Y}_{\xi_{i, j}}(k Y)$ such that $f_{k Y} \sigma_{x}^{n, k} \mathrm{~d} x=\lambda_{n}$ and $\mathfrak{S}_{k Y}\left(\xi_{i, j}, \lambda_{n}\right)=\int_{k Y}\left(\int_{\mathbb{M}} f(x, \zeta) \mathrm{d} \sigma_{x}^{n, k}(\zeta)\right) \mathrm{d} x$. Thus:
$\left(\mathrm{A}_{2}\right) f_{k Y} \sigma_{x}^{n, k} \mathrm{~d} x=\lambda_{n}$ for all $k \geq 1$;
$\left(\mathrm{B}_{2}\right) \lim _{k \rightarrow+\infty} f_{k Y}\left(\int_{\mathbb{M}} f(x, \zeta) \mathrm{d} \sigma_{x}^{n, k}(\zeta)\right) \mathrm{d} x=g\left(\xi_{i, j}, \lambda_{n}\right)$.

For each $n, k \geq 1$ and each $\varepsilon>0$, set $Z_{k, \varepsilon}:=\left\{z \in \mathbb{Z}^{N}: \varepsilon(k Y+z) \subset \Omega_{i, j}\right\}, U_{k, \varepsilon}:=\cup_{z \in Z_{k, \varepsilon}} \varepsilon(k Y+z)$, and define $\left\{\sigma_{x}^{n, k, \varepsilon}\right\}_{x \in \Omega_{i, j}}$ by

$$
\sigma_{x}^{n, k, \varepsilon}:= \begin{cases}\sigma_{\frac{x}{\varepsilon}}^{n, k, \#} & \text { if } x \in U_{k, \varepsilon} \\ \delta_{\xi_{i, j}} & \text { if } x \in \Omega_{i, j} \backslash U_{k, \varepsilon}\end{cases}
$$

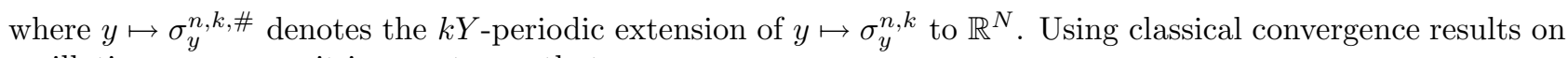
oscillating sequences, it is easy to see that:

$$
\begin{aligned}
& \left(\mathrm{A}_{3}\right) \sigma_{x}^{n, k, \varepsilon} \otimes \mathrm{d} x \stackrel{\text { nar }}{\rightarrow}\left(f_{k Y} \sigma_{y}^{n, k} \mathrm{~d} y\right) \otimes \mathrm{d} x \text { as } \varepsilon \rightarrow 0 \\
& \left(\mathrm{~B}_{3}\right) \lim _{\varepsilon \rightarrow 0} \int_{\Omega_{i, j}}\left(\int_{\mathbb{M}} f\left(\frac{x}{\varepsilon}, \zeta\right) \mathrm{d} \nu_{x}^{n, k, \varepsilon}(\zeta)\right) \mathrm{d} x=\left|\Omega_{i, j}\right| f_{k Y}\left(\int_{\mathbb{M}} f(x, \zeta) \mathrm{d} \sigma_{x}^{n, k}(\zeta)\right) \mathrm{d} x .
\end{aligned}
$$

Since $\sigma_{x}^{n, k} \otimes \mathrm{d} x \in \nabla \mathcal{Y}_{\xi_{i, j}}(k Y)$, there exists a bounded sequence $\left\{u_{\ell}^{n, k}\right\}_{\ell \geq 1} \subset l_{\xi_{i, j}}+W_{0}^{1, p}\left(k Y ; \mathbb{R}^{m}\right)$ such that $\delta_{\nabla u_{\ell}^{n, k}(x)} \otimes \mathrm{d} x \stackrel{\text { nar }}{\longrightarrow} \sigma_{x}^{n, k} \otimes \mathrm{d} x$ as $\ell \rightarrow+\infty$. For each $\ell \geq 1$, define $u_{\ell}^{n, k, \varepsilon} \in l_{\xi_{i, j}}+W_{0}^{1, p}\left(\Omega_{i, j} ; \mathbb{R}^{m}\right)$ by

$$
u_{\ell}^{n, k, \varepsilon}(x):= \begin{cases}u_{\ell}^{n, k, \#}\left(\frac{x}{\varepsilon}\right) & \text { if } x \in U_{k, \varepsilon} \\ l_{\xi_{i, j}}(x) & \text { if } x \in \Omega_{i, j} \backslash U_{k, \varepsilon}\end{cases}
$$

where $u_{\ell}^{n, k, \#}$ denotes the $k Y$-periodic extension of $u_{\ell}^{n, k}$ to $\mathbb{R}^{N}$. An easy computation shows that

$\left(\mathrm{A}_{4}\right) \delta_{\nabla u_{\ell}^{n, k, \varepsilon}(x)} \otimes \mathrm{d} x \stackrel{\text { nar }}{\rightarrow} \sigma_{x}^{n, k, \varepsilon} \otimes \mathrm{d} x$ as $\ell \rightarrow+\infty$. 
By using a truncation argument, we can modify $u_{\ell}^{n, k, \varepsilon}$ (on a $1 / \ell$-neighborhood of $\partial \Omega_{i, j}$ ) in a function $\hat{u}_{\ell}^{n, k, \varepsilon} \in$ $W_{0}^{1, p}\left(\Omega_{i, j} ; \mathbb{R}^{m}\right)$ such that $\nabla \hat{u}_{\ell}^{n, k, \varepsilon}-\nabla u_{\ell}^{n, k, \varepsilon} \rightarrow 0$ in measure on $\Omega_{i, j}$ as $\ell \rightarrow+\infty$. Therefore $\left(\mathrm{A}_{4}\right)$ is satisfied with $u_{\ell}^{n, k, \varepsilon}$ replaced by $\hat{u}_{\ell}^{n, k, \varepsilon}$. We can also assume that the sequence $\left\{\left|\nabla \hat{u}_{\ell}^{n, k, \varepsilon}\right|^{p}\right\}_{\ell \geq 1}$ is uniformly integrable (see [20], Lem. 8.15 p. 151), and so is $\left\{f\left(\cdot / \varepsilon, \nabla \hat{u}_{\ell}^{n, k, \varepsilon}\right)\right\}_{\ell \geq 1}$ by the second inequality in $\left(\mathrm{C}_{2}\right)$. Still denoting $\hat{u}_{\ell}^{n, k, \varepsilon}$ by $u_{\ell}^{n, k, \varepsilon}$, the continuity theorem then gives

$\left(\mathrm{B}_{4}\right) \lim _{\ell \rightarrow+\infty} \int_{\Omega_{i, j}} f\left(\frac{x}{\varepsilon}, \nabla u_{\ell}^{n, k, \varepsilon}(x)\right) \mathrm{d} x=\int_{\Omega_{i, j}}\left(\int_{\mathbb{M}} f\left(\frac{x}{\varepsilon}, \zeta\right) \mathrm{d} \sigma_{x}^{n, k, \varepsilon}(\zeta)\right) \mathrm{d} x$.

Finally, taking $\left(\mathrm{A}_{1}\right),\left(\mathrm{A}_{2}\right),\left(\mathrm{A}_{3}\right)$ and $\left(\mathrm{A}_{4}\right)$ together with $\left(\mathrm{B}_{1}\right),\left(\mathrm{B}_{2}\right),\left(\mathrm{B}_{3}\right)$ and $\left(\mathrm{B}_{4}\right)$ into account, and using standard diagonalization arguments ${ }^{5}$, we can assert that there exist mappings $\varepsilon \mapsto n_{\varepsilon}, \varepsilon \mapsto k_{\varepsilon}$ and $\varepsilon \mapsto \ell_{\varepsilon}$, tending to $+\infty$ as $\varepsilon \rightarrow 0$, such that:

$$
\begin{aligned}
& \left(\mathrm{A}_{i, j}\right) \delta_{\nabla u_{\varepsilon}^{i, j}(x)} \otimes \mathrm{d} x \stackrel{\text { nar }}{\longrightarrow} \mu_{a_{i, j}} \otimes \mathrm{d} x \text { as } \varepsilon \rightarrow 0 ; \\
& \left(\mathrm{B}_{i, j}\right) \lim _{\varepsilon \rightarrow 0} \int_{\Omega_{i, j}} f\left(\frac{x}{\varepsilon}, \nabla u_{\varepsilon}^{i, j}(x)\right) \mathrm{d} x=\left|\Omega_{i, j}\right| \bar{g}\left(\xi_{x_{i, j}}, \mu_{a_{i, j}}\right),
\end{aligned}
$$

where $u_{\varepsilon}^{i, j}:=u_{\ell_{\varepsilon}}^{n_{\varepsilon}, k_{\varepsilon}, \varepsilon}$.

Remark 3.1. Roughly, the parameter $\ell$ accounts for the oscillations of the gradients $\nabla u_{\ell}^{n, k, \varepsilon}$ due to the multiwell structure, the parameter $\varepsilon$ for the oscillations of the corresponding generated Young measure due to the the periodic structure of the material. The parameter $n$ can be seen as a lower semicontinuous regularization parameter.

Step 3 (end of the proof). For each $j, q \geq 1$ and each $\varepsilon>0$, define $u_{q, \varepsilon}^{j} \in \mathcal{U}$ by

$$
u_{q, \varepsilon}^{j}(x):=\left\{\begin{array}{cl}
u_{\varepsilon}^{i, j}(x) & \text { if } x \in \Omega_{i, j} \text { with } i \in\{1, \cdots, q\} \\
0 & \text { if } x \in \Omega \backslash \cup_{i=1}^{q} \Omega_{i, j} .
\end{array}\right.
$$

Then we have

$$
\int_{\Omega} f\left(\frac{x}{\varepsilon}, \nabla u_{q, \varepsilon}^{j}(x)\right) \mathrm{d} x=\sum_{i=1}^{q} \int_{\Omega_{i, j}} f\left(\frac{x}{\varepsilon}, \nabla u_{\varepsilon}^{i, j}(x)\right) \mathrm{d} x+\int_{\Omega \backslash \cup_{i=1}^{q} \Omega_{i, j}} f\left(\frac{x}{\varepsilon}, 0\right) \mathrm{d} x .
$$

But, since $f(\cdot, 0)$ is $Y$-periodic and $\left|\Omega \backslash \cup_{i=1}^{\infty} \Omega_{i, j}\right|=0$,

$$
\lim _{q \rightarrow+\infty} \lim _{\varepsilon \rightarrow 0} \int_{\Omega \backslash \cup_{i=1}^{q} \Omega_{i, j}} f\left(\frac{x}{\varepsilon}, 0\right) \mathrm{d} x=\lim _{q \rightarrow+\infty}\left|\Omega \backslash \cup_{i=1}^{q} \Omega_{i, j}\right| \int_{Y} f(y, 0) \mathrm{d} y=0,
$$

hence by $\left(\mathrm{B}_{i, j}\right)$

$$
\lim _{q \rightarrow+\infty} \lim _{\varepsilon \rightarrow 0} \int_{\Omega} f\left(\frac{x}{\varepsilon}, \nabla u_{q, \varepsilon}^{j}(x)\right) \mathrm{d} x=\sum_{i=1}^{\infty}\left|\Omega_{i, j}\right| \bar{g}\left(\operatorname{bar}\left(\mu_{a_{i, j}}\right), \mu_{a_{i, j}}\right) .
$$

From (21) it follows that

$$
\lim _{j \rightarrow+\infty} \lim _{q \rightarrow+\infty} \lim _{\varepsilon \rightarrow 0} \int_{\Omega} f\left(\frac{x}{\varepsilon}, \nabla u_{q, \varepsilon}^{j}(x)\right) \mathrm{d} x=\int_{\Omega} \bar{g}\left(\operatorname{bar}\left(\mu_{x}\right), \mu_{x}\right) \mathrm{d} x
$$

Similarly, from $\left(\mathrm{A}_{i, j}\right)$ and $(22)$ we obtain

$$
\delta_{\nabla u_{q, \varepsilon}^{j}(x)} \otimes \mathrm{d} x \stackrel{\text { nar }}{\longrightarrow} \mu \text { as first } \varepsilon \rightarrow 0, \text { then } q \rightarrow+\infty \text { and finally } j \rightarrow+\infty .
$$

\footnotetext{
${ }^{5}$ Such arguments are valid because the set $\mathcal{Y}\left(\Omega_{i, j}, \mathbb{M}\right)$ endowed with the narrow topology is metrizable (see [8], Prop. 2.3.1).
} 
Taking (23) together with (24) into account and using a diagonalization argument, we deduce that there exist mappings $\varepsilon \mapsto q_{\varepsilon}$ and $\varepsilon \mapsto j_{\varepsilon}$, tending to $+\infty$ as $\varepsilon \rightarrow 0$, such that:

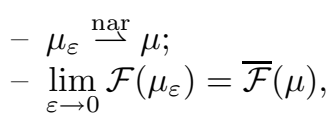

where $\mu_{\varepsilon}:=\delta_{\nabla u_{q \varepsilon}, \varepsilon}^{j_{\varepsilon}(x)}(\mathrm{d} x$, and the proof of the upper bound is complete.

\section{Proof of Theorem 1.4}

Fix $\xi \in \mathbb{M}$. Let $\mu_{x} \otimes \mathrm{d} x \in \nabla \mathcal{Y}(Y)$ be such that $\operatorname{bar}\left(\mu_{x}\right)=\xi$, and let $\left\{\lambda_{n}\right\}_{n \geq 1} \subset \mathcal{P}(\mathbb{M})$ be such that $\lambda_{n} \stackrel{*}{\rightarrow} \mu_{x}$. It is clear that for any $n \geq 1$,

$$
\begin{aligned}
g\left(\xi, \lambda_{n}\right) & \geq \inf _{k \in \mathbb{N}^{*}} \frac{\mathfrak{S}_{k Y}\left(\xi, \lambda_{n}\right)}{k^{N}} \\
& \geq \inf _{k \in \mathbb{N}^{*}} \inf \left\{f_{k Y}\left(\int_{\mathbb{M}} \mathcal{Q} f(x, \zeta) \mathrm{d} \sigma_{x}(\zeta)\right) \mathrm{d} x: \sigma \in \nabla \mathcal{Y}_{\xi}(k Y)\right\} .
\end{aligned}
$$

Moreover, Kinderlehrer-Pedregal's characterization theorem asserts that for every $\sigma \in \nabla \mathcal{Y}_{\xi}(k Y)$, there exists $\phi \in W_{0}^{1, p}\left(\Omega ; \mathbb{R}^{m}\right)$ such that $\int_{\mathbb{M}} \mathcal{Q} f(x, \zeta) \mathrm{d} \sigma_{x}(\zeta) \geq \mathcal{Q} f\left(x, \int_{\mathbb{M}} \zeta \mathrm{d} \sigma_{x}(\zeta)\right)=\mathcal{Q} f(x, \xi+\nabla \phi(x))$ for a.e. $x \in \Omega$, hence

$$
\begin{aligned}
g\left(\xi, \lambda_{n}\right) & \geq \inf _{k \in \mathbb{N}^{*}} \inf \left\{f_{k Y} \mathcal{Q} f(x, \xi+\nabla \phi(x)) \mathrm{d} x: \phi \in W_{0}^{1, p}\left(k Y ; \mathbb{R}^{m}\right)\right\} \\
& =(\mathcal{Q} f)_{\text {hom }}(\xi)=f_{\text {hom }}(\xi) .
\end{aligned}
$$

Letting $n \rightarrow+\infty$, we have $\bar{g}\left(\xi, \mu_{x}\right) \geq \underline{\lim }_{n \rightarrow+\infty} g\left(\xi, \lambda_{n}\right) \geq f_{\text {hom }}(\xi)$, and consequently

$$
\inf \left\{\int_{Y} \bar{g}\left(\xi, \mu_{x}\right) \mathrm{d} x: \mu_{x} \otimes \mathrm{d} x \in \nabla \mathcal{Y}(Y), \operatorname{bar}\left(\mu_{x}\right)=\xi\right\} \geq f_{\text {hom }}(\xi) .
$$

From the homogenization theorem of Braides and Müller, we deduce that there exists a sequence $\left\{u_{\varepsilon}\right\}_{\varepsilon} \subset$ $W^{1, p}\left(Y ; \mathbb{R}^{m}\right)$ such that $u_{\varepsilon} \rightarrow l_{\xi}$ in $W^{1, p}\left(Y ; \mathbb{R}^{m}\right)$ and

$$
\lim _{\varepsilon \rightarrow 0} \int_{Y} f\left(\frac{x}{\varepsilon}, \nabla u_{\varepsilon}(x)\right) \mathrm{d} x=f_{\text {hom }}(\xi) .
$$

Using Prokorov's compactness theorem (cf. Rem. 2.1) together with the continuity theorem, we obtain the existence of $\mu_{x} \otimes \mathrm{d} x \in \nabla \mathcal{Y}(Y)$ such that $\operatorname{bar}\left(\mu_{x}\right)=\xi$ for a.e. $x \in \Omega$ and (up to a subsequence) $\delta_{\nabla u_{\varepsilon}(x)} \otimes \mathrm{d} x \stackrel{\text { nar }}{\longrightarrow}$ $\mu_{x} \otimes \mathrm{d} x$. By (25) and Theorem 1.1, it follows that

$$
\int_{Y} \bar{g}\left(\xi, \mu_{x}\right) \mathrm{d} x \leq \varliminf_{\varepsilon \rightarrow 0} \int_{Y} f\left(\frac{x}{\varepsilon}, \nabla u_{\varepsilon}(x)\right) \mathrm{d} x=f_{\text {hom }}(\xi) .
$$

Thus

$$
\inf \left\{\int_{Y} \bar{g}\left(\xi, \mu_{x}\right) \mathrm{d} x: \mu_{x} \otimes \mathrm{d} x \in \nabla \mathcal{Y}(Y), \operatorname{bar}\left(\mu_{x}\right)=\xi\right\} \leq f_{\text {hom }}(\xi),
$$

and the proof of Theorem 1.4 is complete. 


\section{EXTENSIONS AND OPEN QUESTIONS}

\subsection{Extension to the stochastic case}

Let $\mathcal{I}$ denote the class of all Carathéodory integrands $w: \mathbb{R}^{N} \times \mathbb{M} \rightarrow \mathbb{R}$ such that $\alpha|\xi|^{p} \leq w(x, \xi) \leq \beta\left(1+|\xi|^{p}\right)$ for all $x \in \mathbb{R}^{N}$, all $\xi \in \mathbb{M}$ and some $\alpha, \beta>0$. Let $(\Sigma, \mathfrak{T}, \mathbf{P})$ be a probability space and let $f: \Sigma \times \mathbb{R}^{N} \times \mathbb{M} \rightarrow \mathbb{R}$ be a measurable function such that

$$
f(\omega, \cdot, \cdot) \in \mathcal{I} \text { for all } \omega \in \Sigma .
$$

Such a $f$ is called a random integrand: when $m=N=3$, it can be interpreted as the free-energy density of a randomly heterogeneous material. Consider $\mathfrak{I}$ the trace on $\mathcal{I}$ of the product $\sigma$-algebra of $\mathbb{R}^{\mathbb{R}^{N} \times \mathbb{M}}$, and, for each $z \in \mathbb{Z}^{N}$, define $\tau_{z}: \mathcal{I} \rightarrow \mathcal{I}$ by

$$
\left(\tau_{z} w\right)(x, \xi):=w(x+z, \xi)
$$

Then, $\left\{\tau_{z}\right\}_{z \in \mathbb{Z}^{N}}$ is an additive group of measurable transformations on $(\mathcal{I}, \mathfrak{I})$. Let $f_{\#} \mathbf{P}$ denote the image of the probability $\mathbf{P}$ by the measurable map $\Sigma \ni \omega \mapsto f(\omega, \cdot, \cdot) \in \mathcal{I}$. The $Y$-periodicity assumption (corresponding to the deterministic case) is replaced by the following:

$\left(\mathrm{H}_{1}\right) f$ is periodic in law, i.e., $f_{\#} \mathbf{P}(E)=f_{\#} \mathbf{P}\left(\tau_{z}(E)\right)$ for all $z \in \mathbb{Z}^{N}$ and all $E \in \mathfrak{I}$.

For every $\varepsilon>0$, we define $\mathcal{F}_{\varepsilon}: \Sigma \times \mathcal{Y}(\Omega ; \mathbb{M}) \rightarrow[0,+\infty]$ by

$$
\mathcal{F}_{\varepsilon}(\omega, \mu):=\left\{\begin{array}{c}
\int_{\Omega}\left(\int_{\mathbb{M}} f\left(\omega, \frac{x}{\varepsilon}, \xi\right) \mathrm{d} \mu_{x}(\xi)\right) \mathrm{d} x \text { if } \mu=\mu_{x} \otimes \mathrm{d} x \in \Delta(\mathcal{U}) \\
+\infty
\end{array}\right.
$$

In order that the $\Gamma$ (nar)-limit of $\left\{\mathcal{F}_{\varepsilon}(\omega, \cdot)\right\}_{\varepsilon}$ does not depend on $\omega$, it is usual to make the following assumption (see $[12,17]$ for more details):

$\left(\mathrm{H}_{2}\right) f$ is ergodic, i.e., $f_{\#} \mathbf{P}(E) \in\{0,1\}$ whenever $E \in \mathfrak{I}$ is $\tau$-invariant $\left(\tau_{z}(E)=E\right.$ for all $\left.z \in \mathbb{Z}^{N}\right)$.

For every $A \in \mathcal{O}_{b}$, we consider $\mathfrak{S}_{A}: \mathcal{I} \times \mathbb{M} \times \mathcal{P}(\mathbb{M}) \rightarrow[0,+\infty]$ given by

$$
\mathfrak{S}_{A}(w, \xi, \lambda):=\inf \left\{\int_{A}\left(\int_{\mathbb{M}} w(x, \zeta) \mathrm{d} \sigma_{x}(\zeta)\right) \mathrm{d} x: \sigma_{x} \otimes \mathrm{d} x \in \Gamma_{A}(\xi, \lambda)\right\}
$$

with $\Gamma_{A}: \mathbb{M} \times \mathcal{P}(\mathbb{M}) \longrightarrow \mathcal{Y}(A ; \mathbb{M})$ defined by (7). Taking (8) into account, we see that for every $w \in \mathcal{I}$, $\mathfrak{S}_{A}(w, \xi, \lambda)=+\infty$ if and only if $\lambda \notin \mathcal{H}_{\xi}(\mathbb{M})$. For $\lambda \in \mathcal{H}_{\xi}(\mathbb{M})$ with $\xi \in \mathbb{M}$, it is clear that

$$
\mathfrak{S}_{A}(w, \xi, \lambda) \leq \beta\left(1+\int_{\mathbb{M}}|\zeta|^{p} \mathrm{~d} \lambda(\zeta)\right)|A| \text { for all } A \in \mathcal{O}_{b} \text { and all } w \in \mathcal{I}
$$

Condition $\left(\mathrm{H}_{1}\right)$ implies that $\mathfrak{S}_{(\cdot)}(\cdot, \xi, \lambda)$ is $\tau$-covariant, i.e.,

$$
\mathfrak{S}_{A}\left(\tau_{z} w, \xi, \lambda\right)=\mathfrak{S}_{A+z}(w, \xi, \lambda) \text { for all } z \in \mathbb{Z}^{N} \text {, all } A \in \mathcal{O}_{b} \text { and all } w \in \mathcal{I}
$$

and $\left(\mathrm{H}_{2}\right)$ exactly means that the group $\left\{\tau_{z}\right\}_{z \in \mathbb{Z}^{N}}$ is ergodic on the probability space $\left(\mathcal{I}, \mathfrak{I}, f_{\#} \mathbf{P}\right)$. The set function $\mathfrak{S}_{(\cdot)}(w, \xi, \lambda)$ being subadditive for each $w \in \mathcal{I}$, as a consequence of Akcoglu-Krengel's subadditive ergodic theorem, we obtain the following "ergodic version" of Proposition 2.4.

Proposition 2.4'. Given $\xi \in \mathbb{M}$ and $\lambda \in \mathcal{H}_{\xi}(\mathbb{M})$, if $\left(\mathrm{H}_{1}\right)$ and $\left(\mathrm{H}_{2}\right)$ hold then for $f_{\#} \mathbf{P}$-a.e. $w \in \mathcal{I}$,

$$
\lim _{k \rightarrow+\infty} \frac{\mathfrak{S}_{A}(w, \xi, \lambda)}{k^{N}}=\inf _{k \in \mathbb{N}^{*}} \frac{\mathbf{E S}_{k Y}(\cdot, \xi, \lambda)}{k^{N}}=\inf _{k \in \mathbb{N}^{*}} \frac{1}{k^{N}} \int_{\Sigma} \mathfrak{S}_{k Y}(f(\omega, \cdot, \cdot), \xi, \lambda) \mathrm{d} \mathbf{P}(\omega),
$$

where $\mathbf{E}$ denotes the expectation operator with respect to $f_{\#} \mathbf{P}$. 
Proposition $2.4^{\prime}$ leads us to define $g: \mathbb{M} \times \mathcal{P}(\mathbb{M}) \rightarrow[0,+\infty]$ by

$$
g(\xi, \lambda):=\left\{\begin{array}{cc}
\inf _{k \in \mathbb{N}^{*}} \frac{1}{k^{N}} \int_{\Sigma} \mathfrak{S}_{k Y}(f(\omega, \cdot, \cdot), \xi, \lambda) \mathrm{d} \mathbf{P}(\omega) & \text { if } \lambda \in \mathcal{H}_{\xi}(\mathbb{M}) \\
+\infty & \text { otherwise }
\end{array}\right.
$$

Then, similarly to the deterministic case, we consider $\bar{g}: \mathbb{M} \times \mathcal{P}(\mathbb{M}) \rightarrow[0,+\infty]$ given by

$$
\bar{g}(\xi, \lambda):=\inf \left\{\varliminf_{n \rightarrow+\infty} g\left(\xi, \lambda_{n}\right): \mathcal{P}(\mathbb{M}) \ni \lambda_{n} \stackrel{*}{\rightarrow} \lambda\right\}
$$

and we define $\overline{\mathcal{F}}: \mathcal{Y}(\Omega ; \mathbb{M}) \rightarrow[0,+\infty]$ by

$$
\overline{\mathcal{F}}(\mu):=\left\{\begin{array}{cl}
\int_{\Omega} \bar{g}\left(\operatorname{bar}\left(\mu_{x}\right), \mu_{x}\right) \mathrm{d} x & \text { if } \mu=\mu_{x} \otimes \mathrm{d} x \in \mathfrak{U} \\
+\infty & \text { otherwise. }
\end{array}\right.
$$

In our opinion, it is not difficult to extend Theorem 1.1 to the stochastic case as follows.

Conjecture 5.1. Under $\left(\mathrm{H}_{1}\right)$ and $\left(\mathrm{H}_{2}\right), \Gamma(\operatorname{nar})-\lim _{\varepsilon \rightarrow 0} \mathcal{F}_{\varepsilon}(\omega,)=.\overline{\mathcal{F}}$ for $\mathbf{P}$-a.e. $\omega \in \Sigma$.

\subsection{Toward the analysis of oscillations-concentrations}

The gradient Young measure associated with a bounded sequence $\left\{u_{\varepsilon}\right\}_{\varepsilon}$ in $W^{1, p}\left(\Omega ; \mathbb{R}^{m}\right)$ is convenient to describe the oscillations of $\left\{\nabla u_{\varepsilon}\right\}_{\varepsilon}$. On the other hand, the effects of concentrations are completely missed by this tool. Indeed, any $\left\{v_{\varepsilon}\right\}_{\varepsilon}$, with $v_{\varepsilon} \in W_{0}^{1, p}\left(\Omega ; \mathbb{R}^{m}\right)$ and $\nabla u_{\varepsilon}-\nabla v_{\varepsilon} \rightarrow 0$ in measure on $\Omega$ as $\varepsilon \rightarrow 0$, generates the same gradient Young measure. Thus, the (possible) concentrations, for example on $\partial \Omega$, cannot be characterized in this way. In fact, to account for the development of concentrations, we need the notion of $W^{1, p}$-varifold introduced by Fonseca, Müller and Pedregal [14]. We are thus led to consider another formulation of the functional $F_{\varepsilon}$ in (1) in terms of Young measures-varifolds.

Let $\mathcal{M}^{+}(\bar{\Omega} \times \mathbb{S})$ be the set of all positive Radon measures on $\bar{\Omega} \times \mathbb{S}$, where $\mathbb{S}$ is the unit sphere in $\mathbb{M}$. Since concentration phenomena are related to the behavior of $f(x, \cdot)$ at infinity, we make the following assumption:

$\left(\mathrm{C}_{3}\right)$ there exists a function $f^{\infty}: \mathbb{R}^{N} \times \mathbb{M} \rightarrow\left[0,+\infty\left[\right.\right.$ such that for every $x \in \mathbb{R}^{N}$,

$$
\lim _{|\xi| \rightarrow+\infty} \frac{1}{|\xi|^{p}}\left(f(x, \xi)-f^{\infty}(x, \xi)\right)=0
$$

and, for each $\varepsilon>0$, we consider $\mathcal{F}_{\varepsilon}: \mathcal{Y}(\Omega ; \mathbb{M}) \times \mathcal{M}^{+}(\bar{\Omega} \times \mathbb{S}) \rightarrow[0,+\infty]$ defined by

$$
\mathcal{F}_{\varepsilon}(\mu, \nu):=\left\{\begin{array}{c}
\int_{\Omega \times \mathbb{M}}\left[f-f^{\infty}\right]\left(\frac{x}{\varepsilon}, \xi\right) \mathrm{d} \mu+\int_{\bar{\Omega} \times \mathbb{S}} f_{+\infty}^{\infty}\left(\frac{x}{\varepsilon}, \xi\right) \mathrm{d} \nu \text { if }(\mu, \nu) \in \Delta(\mathcal{U}) \\
\text { otherwise }
\end{array}\right.
$$

with

$$
\Delta(\mathcal{U}):=\left\{\left(\delta_{\widetilde{\nabla} u(x)} \otimes \mathrm{d} x, \delta_{\frac{\nabla u}{|\nabla u|}(x)} \otimes|\widetilde{\nabla u}|^{p} \mathrm{~d} x\right): u \in \mathcal{U}\right\}
$$


where $\widetilde{\nabla u}$ is the zero extension of $\nabla u$ to $\mathbb{R}^{N} \backslash \Omega$. Denoting by $\mathfrak{U}$ the set of all $(\mu, \nu) \in \mathcal{Y}(\Omega ; \mathbb{M}) \times \mathcal{M}^{+}(\bar{\Omega} \times \mathbb{S})$ such that:

- $\mu$ is a $\mathcal{U}$-gradient Young measure on $\Omega \times \mathbb{M}$;

- $\nu$ is a $\mathcal{U}$-varifold on $\bar{\Omega} \times \mathbb{S}$, i.e., there exists a bounded sequence $\left\{u_{\varepsilon}\right\}_{\varepsilon}$ in $\mathcal{U}$ such that $\nu$ is the weak* limit of $\delta_{\frac{\nabla u_{\varepsilon}}{\left|\nabla u_{\varepsilon}\right|}(x)} \otimes\left|\widetilde{\nabla u_{\varepsilon}}\right|^{p} \mathrm{~d} x$ as $\varepsilon \rightarrow 0$,

it seems to us reasonable to make the following conjecture that we hope to study in a future work.

Conjecture 5.2. Under $\left(\mathrm{C}_{1}\right),\left(\mathrm{C}_{2}\right)$ and $\left(\mathrm{C}_{3}\right)$, we have ${ }^{6} \Gamma(\mathrm{nar}, *)-\lim _{\varepsilon \rightarrow 0} \mathcal{F}_{\varepsilon}=\overline{\mathcal{F}}$ with $\overline{\mathcal{F}}: \mathcal{Y}\left(\Omega ; \mathbb{M}^{2} \times \mathcal{M}^{+}(\bar{\Omega} \times\right.$ $\mathbb{S}) \rightarrow[0,+\infty]$ of the form:

$$
\overline{\mathcal{F}}(\mu, \nu)=\left\{\begin{array}{cl}
\int_{\Omega} \bar{f}_{1}\left(\operatorname{bar}\left(\mu_{x}\right), \mu_{x}, \frac{\mathrm{d} \pi}{\mathrm{d} x} \nu_{x}\right) \mathrm{d} x+\int_{{ }_{\bar{\Omega}}} \bar{f}_{2}\left(\operatorname{bar}\left(\nu_{x}\right), \nu_{x}\right) \mathrm{d} \pi^{s} & \text { if }(\mu, \nu) \in \mathfrak{U} \\
\text { otherwise },
\end{array}\right.
$$

where $\bar{f}_{1}: \mathbb{M} \times \mathcal{P}(\mathbb{M}) \times \mathcal{M}^{+}(\mathbb{S}) \rightarrow\left[0,+\infty\left[, \bar{f}_{2}: \mathbb{S} \times \mathcal{P}(\mathbb{S}) \rightarrow\left[0,+\infty\left[, \pi\right.\right.\right.\right.$ is a positive measure on $\bar{\Omega}, \nu=\nu_{x} \otimes \pi$, $\operatorname{bar}\left(\nu_{x}\right):=\int_{\mathbb{S}} \zeta \mathrm{d} \nu_{x}(\zeta)$ and $\pi=\frac{\mathrm{d} \pi}{\mathrm{d} x} \mathrm{~d} x+\pi_{s}$ is the Radon-Nikodym decomposition of $\pi$.

\section{REFERENCES}

[1] M.A. Akcoglu and U. Krengel, Ergodic theorems for superadditive processes. J. Reine Angew. Math. 323 (1981) 53-67.

[2] F. Alvarez and J.-P. Mandallena, Homogenization of multiparameter integrals. Nonlinear Anal. 50 (2002) 839-870.

[3] H. Attouch, Variational convergence for functions and operators. Pitman (1984).

[4] J.M. Ball and R.D. James, Fine phase mixtures as minimizers of energy. Arch. Rat. Mech. Anal. 100 (1987) 13-52.

[5] K. Bhattacharya and R. Kohn, Elastic energy minimization and the recoverable strains of polycristalline shape-memory materials. Arch. Rat. Mech. Anal. 139 (1997) 99-180.

[6] A. Braides, Homogenization of some almost periodic coercive functional. Rend. Accad. Naz. Sci. 103 (1985) $313-322$.

[7] A. Braides and A. Defranceschi, Homogenization of multiple integrals. Oxford University Press (1998).

[8] C. Castaing, P. Raynaud de Fitte and M. Valadier, Young measures on topological spaces with applications in control theory and probability theory. Mathematics and Its Applications, Kluwer, The Netherlands (2004).

[9] C. Castaing and M. Valadier, Convex analysis and measurable multifunctions. Lect. Notes Math. 580 (1977).

[10] B. Dacorogna, Quasiconvexity and relaxation of nonconvex variational problems. J. Funct. Anal. 46 (1982) 102-118.

[11] G. Dal maso, An introduction to $\Gamma$-convergence. Birkhäuser (1993).

[12] G. Dal maso and L. Modica, Nonlinear stochastic homogenization. J. Reine Angew. Math. 363 (1986) $27-43$.

[13] L.C. Evans, Weak convergence methods for nonlinear partial differential equations. CBMS Amer. Math. Soc. 74 (1990).

[14] I. Fonseca, S. Müller and P. Pedregal, Analysis of concentration and oscillation effects generated by gradients. SIAM J. Math. Anal. 29 (1998) 736-756.

[15] D. Kinderlherer and P. Pedregal, Characterization of Young measure generated by gradients. Arch. Rat. Mech. Anal. 115 (1991) 329-365.

[16] D. Kinderlherer and P. Pedregal, Gradient Young measures generated by sequences in Sobolev spaces. J. Geom. Anal. 4 (1994) 59-89.

[17] C. Licht and G. Michaille, Global-local subadditive ergodic theorems and application to homogenization in elasticity. Ann. Math. Blaise Pascal 9 (2002) 21-62.

[18] P. Marcellini, Periodic solutions and homogenization of nonlinear variational problems. Annali Mat. Pura Appl. 117 (1978) 139-152.

[19] S. Müller, Homogenization of nonconvex integral functionals and cellular elastic materials. Arch. Rat. Mech. Anal. 100 (1987) 189-212.

[20] P. Pedregal, Parametrized measures and variational principles. Birkhäuser (1997).

[21] P. Pedregal, Г-convergence through Young meaasures. SIAM J. Math. Anal. 36 (2004) 423-440.

[22] M. Valadier, Young measures. Lect. Notes Math. 1446 (1990) 152-188.

[23] M. Valadier, A course on Young measures. Rend. Istit. Mat. Univ. Trieste 26 (1994) Suppl. 349-394.

[24] W.P. Ziemer, Weakly differentiable functions. Springer (1989).

\footnotetext{
${ }^{6}$ Here the $\Gamma$-convergence is taken with respect to the product of the narrow convergence in $\mathcal{Y}(\Omega ; \mathbb{M})$ by the weak* convergence in $\mathcal{M}^{+}(\bar{\Omega} \times \mathbb{S})$.
} 